\title{
The Method of Unitary Clothing Transformations in Quantum Field Theory: Applications in the Theory of Nuclear Forces and Reactions
}

\author{
A. V. Shebeko \\ Institute for Theoretical Physics \\ National Research Center "Kharkov Institute of Physics E Technology"
}

Ukraine

\section{Introduction}

In what follows we will show how one can realize the notion of "clothed " particles (Greenberg \& Schweber, 1958) for field theoretical treatments based upon the so-called instant form of relativistic dynamics formulated by Dirac (Dirac, 1949). In the context, let us recall that the notion points out a transparent way for including the so-called cloud or persistent effects in a system of interacting fields (to be definite, mesons and nucleons). A constructive step (see surveys (Shebeko \& Shirokov, 2000; 2001) and refs. therein) is to express the total field Hamiltonian $H$ and other operators of great physical meaning, e.g., the Lorentz boost generators and current density operators, which depend initially on the creation and destruction operators for the "bare" particles, through a set of their "clothed" counterparts. It is achieved via unitary clothing transformations (UCTs) (see article (Korda et al., 2007)) in the Hilbert space $\mathcal{H}$ of meson-nucleon states and we stress, as before, that each of such transformations remains the Hamiltonian unchanged unlike other unitary transformation methods (Glöckle \& Müller, 1981; Kobayashi, 1997; Okubo, 1954; Stefanovich, 2001)) ${ }^{1}$ for Hamiltonian-based models. In the course of the clothing procedure a large amount of virtual processes associated in our case with the meson absorption/emission, the $N \bar{N}$-pair annihilation/production and other cloud effects turns out to be accumulated in the creation (destruction) operators for the clothed particles. The latter, being the quasiparticles of the method of UCTs, must have the properties (charges, masses, etc.) of physical (observable) particles. Such a bootstrap reflects the most significant distinction between the concepts of clothed and bare particles.

At the same time, after Dirac, any relativistic quantum theory may be so defined that the generator of time translations (Hamiltonian), the generators of space translations (linear momentum), space rotations (angular momentum) and Lorentz transformations (boost operator) satisfy the well-known commutations. Basic ideas, put forward by Dirac with his "front", "instant" and "point" forms of the relativistic dynamics, have been realized in many relativistic quantum mechanical models. In this context, the survey (Keister \& Polyzou, 1991), being a remarkable introduction to a subfield called the relativistic Hamiltonian dynamics,

\footnotetext{
${ }^{1}$ Some specific features of these methods are discussed in (Shebeko \& Shirokov, 2001) and (Korda et al., 2007)
} 
represents various aspects and achievements of relativistic direct interaction theories. Among the vast literature on this subject we would like to note an exhaustive exposition in lectures (Bakker, 2001; Heinzl, 2001) of the appealing features of the relativistic Hamiltonian dynamics with an emphasis on "light-cone quantization". Following a pioneering work (Foldy, 1961), the term "direct" is related to a system with a fixed number of interacting particles, where interactions are rather direct than mediated through a field. In the approach it is customary to consider such interactions expressed in terms of the particle coordinates, momenta and spins. Along the guideline the so-called separable interactions and relativistic center-of-mass variables for composite systems were built up by assuming that the generators of the Poincare group $(\Pi)$ can be represented as expansions on powers of $1 / c^{2}$ or, more exactly, $(v / c)^{2}$, where $v$ is a typical nuclear velocity (cf. the $(p / m)$ expansion, introduced in (Friar, 1975), where $m$ is the nucleon mass and $p$ is a typical nucleon momentum). Afterwards, similar expansions were rederived and reexamined (with new physical inputs) in the framework of a field-theoretic approach (Glöckle \& Müller, 1981). There, starting from a model Lagrangian for "scalar nucleons" interacting with a scalar meson field (like the Wentzel model (Wentzel, 1949)) the authors showed (to our knowledge first) how the Hamiltonian and the boost generator (these noncommuting operators), determined in a standard manner (Schwinger, 1962), can be blockdiagonalized by one and the same unitary transformation after Okubo (Okubo, 1954). The corresponding blocks derived in leading order in the coupling constant act in the subspace with a fixed nucleon number (the nucleon "sector" of the full space $\mathcal{H}$ ). In general, the work (Glöckle \& Müller, 1981) and its continuation (Krüger \& Glöckle, 1999) exemplify applications of local relativistic quantum field theory (RQFT), where the generators of interest, being compatible with the basic commutation rules for fields, are constructed within the Lagrangian formalism using the Nöther theorem and its consequences. Although the available covariant perturbation theory and functional-integral methods are very successful when describing various relativistic and quantum effects in the world of elementary particles, the Hamilton method can be helpful too. As known, it is the case, where one has to study properties of strongly interacting particles, e.g., as in nuclear physics with its problems of bound states for meson-nucleon systems. Of course, any Hamiltonian formulation of field theory, not being manifestly covariant, cannot be $a b$ initio accepted as equivalent to the way after Feynman, Schwinger and Tomonaga. However, in order to overcome the obstacle starting from a field Hamiltonian $H$ one can consider it as one of the ten infinitesimal operators (generators) of space-time translations and pure Lorentz transformations that act in a proper Hilbert space. Taken together they compose a basis of the Lie-Poincare algebra (see below) to ensure relativistic invariance (RI) in the Dirac sense, being referred to the RI as a whole.

The purpose of the present exposition is twofold. First, we consider an algebraic method (Shebeko \& Frolov , 2011) to meet the Poincaré commutators for a wide class of field theoretic models (local and nonlocal ones taking into account their invariance with respect to space translations). In particular, this recursive method is appropriate for models with derivative couplings and spins $\geq 1$, typical of the meson theory of nuclear forces, where only some part of the interaction density in the Dirac picture has the property to be a Lorentz scalar. The antiparticle degrees of freedom are included together with such an important issue as mass renormalization vs relativistic invariance in the Dirac sense. Second, special attention is paid to finding analytic expressions for the generators in the clothed-particle representation, in which the so-called bad terms are simultaneously removed from the Hamiltonian and the boosts. Moreover, the mass renormalization terms introduced in the Hamiltonian at the outset 
turn out to be related to certain covariant integrals that are convergent in the field models with proper cutoff factors. After constructing interactions between the clothed particles and addressing an equivalence theorem for evaluation of the $S$-matrix we derive the approximate eigenvalue equations for the simplest bound and scattering states. The latter can be found in a nonperturbative way using the methods elaborated in the theory of nuclear structure and reactions that is demonstrated by a few examples.

However, before to apply the UCT method (in particular, beyond the Lagrangian formalism with its local interaction densities) we would like to mention the two algebraic procedures to solve the basic commutator equations of $\Pi$ (see Sec. 2). One of them, proposed in (Shebeko \& Frolov , 2011), has some touching points with the other developed in (Kita, 1966; 1968) and essentially repeated many years after by Chandler (Chandler, 2003). In paper (Kita, 1968) the author considers three kinds of neutral spinless bosons and nonlocal interaction between them in a relativistic version of the Lee model with a cutoff in momentum space. A similar model for two spinless particles has been utilized in (Chandler, 2003) with a Yukawa-type interaction that belongs to the realm of the so-called models with persistent vacuum (see, for instance, (Eckmann, 1970)). Certain resemblance between our and those explorations is that we prefer to proceed within a corpuscular picture (see Chapter IV in monograph (Weinberg, 1995)), where each of the ten generators of the Poincare group $\Pi$ (and not only they) may be expressed as a sum of products of particle creation and annihilation operators $a^{\dagger}(n)$ and $a(n)$ $(n=1,2, \ldots)$, e.g., bosons and/or fermions. Some mathematical aspects of the corpuscular notion were formulated many years ago in (Friedrichs, 1953) (Chapter III). As in (Weinberg, 1995), a label $n$ is associated with all the necessary quantum numbers for a single particle: its momentum $\mathbf{p}_{n}{ }^{2}$, spin $z$-component (helicity for massless particles) $\mu_{n}$, and species $\xi_{n}$. The operators $a^{\dagger}(n)$ and $a(n)$ satisfy the standard commutation relations such as Eqs. (4.2.5)-(4.2.7) in (Weinberg, 1995).

In the framework of such a picture the Hamiltonian of a system of interacting mesons and nucleons can be written as

$$
\begin{gathered}
H=\sum_{C=0}^{\infty} \sum_{A=0}^{\infty} H_{C A}, \\
H_{C A}=\sum H_{C A}\left(1^{\prime}, 2^{\prime}, \ldots, n_{C}^{\prime} ; 1,2, \ldots, n_{A}\right) a^{\dagger}\left(1^{\prime}\right) a^{\dagger}\left(2^{\prime}\right) \ldots a^{\dagger}\left(n_{C}^{\prime}\right) a\left(n_{A}\right) \ldots a(2) a(1),
\end{gathered}
$$

where the capital $C(A)$ denotes the particle-creation (annihilation) number for the operator substructure $H_{C A}$. Sometimes we say that the latter belongs to the class $[C . A]$. Operation $\mathcal{L}$ implies all necessary summations over discrete indices and covariant integrations over continuous spectra.

Further, it is proved (Weinberg, 1995) that the $S$-matrix meets the so-called cluster decomposition principle, if the coefficient functions $H_{C A}$ embody a single three-dimensional momentum-conservation delta function, viz.,

$$
\begin{gathered}
H_{C A}\left(1^{\prime}, 2^{\prime}, \ldots, C ; 1,2, \ldots, A\right)=\delta\left(\mathbf{p}_{1}^{\prime}+\mathbf{p}_{2}^{\prime}+\ldots+\mathbf{p}_{C}^{\prime}-\mathbf{p}_{1}-\mathbf{p}_{2}-\ldots-\mathbf{p}_{A}\right) \\
\times h_{C A}\left(p_{1}^{\prime} \mu_{1}^{\prime} \xi_{1}^{\prime}, p_{2}^{\prime} \mu_{2}^{\prime} \xi_{2}^{\prime}, \ldots, p_{C}^{\prime} \mu_{C}^{\prime} \xi_{C}^{\prime} ; p_{1} \mu_{1} \xi_{1}, p_{2} \mu_{2} \xi_{2}, \ldots, p_{A} \mu_{A} \xi_{A}\right),
\end{gathered}
$$

\footnotetext{
${ }^{2}$ Or the 4-momentum $p_{n}=\left(p_{n}^{0}, \mathbf{p}_{n}\right)$ on the mass shell $p_{n}^{2}=p_{n}^{02}-\mathbf{p}_{n}^{2}=m_{n}^{2}$ with the particle mass $m_{n}$
} 
where the $c$-number coefficients $h_{C A}$ do not contain delta function.

Following the guideline "to free ourselves from any dependence on pre-existing field theories "(cit. from (Weinberg, 1995) on p.175), the three boost operators $\mathbf{N}=\left(N^{1}, N^{2}, N^{3}\right)$ can be written as

$$
\begin{gathered}
\mathbf{N}=\sum_{C=0}^{\infty} \sum_{A=0}^{\infty} \mathbf{N}_{C A}, \\
\mathbf{N}_{C A}=\sum \mathbf{N}_{C A}\left(1^{\prime}, 2^{\prime}, \ldots, n_{C}^{\prime} ; 1,2, \ldots, n_{A}\right) a^{\dagger}\left(1^{\prime}\right) a^{\dagger}\left(2^{\prime}\right) \ldots a^{\dagger}\left(n_{C}^{\prime}\right) a\left(n_{A}\right) \ldots a(2) a(1) .
\end{gathered}
$$

In turn, the operator $H$, being divided into the no-interaction part $H_{F}$ and the interaction $H_{I}$, owing to its translational invariance allows $H_{I}$ to be written as

$$
H_{I}=\int H_{I}(\mathbf{x}) d \mathbf{x}
$$

Our consideration is focused upon various field models (local and nonlocal) in which the interaction density $H_{I}(\mathbf{x})$ consists of scalar $H_{s c}(\mathbf{x})$ and nonscalar $H_{n s c}(\mathbf{x})$ contributions,

$$
H_{I}(\mathbf{x})=H_{s c}(\mathbf{x})+H_{n s c}(\mathbf{x}),
$$

where the property to be a scalar means

$$
U_{F}(\Lambda) H_{s c}(x) U_{F}^{-1}=H_{s c}(\Lambda x), \quad \forall x=(t, \mathbf{x})
$$

for all Lorentz transformations $\Lambda$. Henceforth, for any operator $O(\mathbf{x})$ in the Schrödinger (S) picture it is introduced its counterpart $O(x)=\exp \left(i H_{F} t\right) O(\mathbf{x}) \exp \left(-i H_{F} t\right)$ in the Dirac (D) picture.

\section{Basic equations in relativistic theory with particle creation and annihilation}

When seeking links between the coefficients in the r.h.s. of Eqs. (2) and (5) one considers the fundamental relations of the Lie-Poincare algebra, which can be divided into the three kinds for:

no-interaction generators

$$
\left[P_{i}, P_{j}\right]=0, \quad\left[J_{i}, J_{j}\right]=i \varepsilon_{i j k} J_{k}, \quad\left[J_{i}, P_{j}\right]=i \varepsilon_{i j k} P_{k},
$$

relations linear in $H$ and $\mathbf{N}$

$$
[\mathbf{P}, H]=0, \quad[\mathbf{J}, H]=0, \quad\left[J_{i}, N_{j}\right]=i \varepsilon_{i j k} N_{k}, \quad\left[P_{i}, N_{j}\right]=i \delta_{i j} H,
$$

and ones nonlinear in $H$ and $\mathbf{N}$

$$
\begin{gathered}
{[H, \mathbf{N}]=i \mathbf{P}, \quad\left[N_{i}, N_{j}\right]=-i \varepsilon_{i j k} J_{k},} \\
(i, j, k=1,2,3),
\end{gathered}
$$

where $\mathbf{P}=\left(P^{1}, P^{2}, P^{3}\right)$ and $\mathbf{J}=\left(J^{1}, J^{2}, J^{3}\right)$ are the linear momentum and angular momentum operators, respectively. In this context, let us remind that in the instant form of relativistic dynamics after Dirac (Dirac, 1949) only the Hamiltonian and the boost operators carry 
interactions with conventional partitions $H=H_{F}+H_{I}$ and $\mathbf{N}=\mathbf{N}_{F}+\mathbf{N}_{I}$, while $\mathbf{P}=\mathbf{P}_{F}$ and $\mathbf{J}=\mathbf{J}_{F}$. In short notations, we distinguish the set $G_{F}=\left\{H_{F}, \mathbf{P}_{F}, \mathbf{J}_{F}, \mathbf{N}_{F}\right\}$ for free particles and the set $G=\left\{H, \mathbf{P}_{F}, \mathbf{J}_{F}, \mathbf{N}\right\}$ for interacting particles.

In turn, every operator $H_{C A}$ can be represented as $H_{C A}=\int H_{C A}(\mathbf{x}) d \mathbf{x}$, if one uses the formula

$$
\delta\left(\mathbf{p}-\mathbf{p}^{\prime}\right)=\frac{1}{(2 \pi)^{3}} \int e^{i\left(\mathbf{p}-\mathbf{p}^{\prime}\right) \mathbf{x}} d \mathbf{x}
$$

Thus, we come to the form $H=\int H(\mathbf{x}) d \mathbf{x}$ well known from local field models with the density

$$
H(\mathbf{x})=\sum_{C=0}^{\infty} \sum_{A=0}^{\infty} H_{C A}(\mathbf{x}) .
$$

For instance, in case with $C=A=2$, where $H_{22}\left(1^{\prime}, 2^{\prime} ; 1,2\right)=\delta\left(\mathbf{p}_{1}^{\prime}+\mathbf{p}_{2}^{\prime}-\mathbf{p}_{1}-\right.$ $\left.\mathbf{p}_{2}\right) h\left(1^{\prime}, 2^{\prime} ; 1,2\right)$, we have

$$
H_{22}(\mathbf{x})=\frac{1}{(2 \pi)^{3}} \sum \exp \left[-i\left(\mathbf{p}_{1}^{\prime}+\mathbf{p}_{2}^{\prime}-\mathbf{p}_{1}-\mathbf{p}_{2}\right) \mathbf{x}\right] h\left(1^{\prime}, 2^{\prime} ; 1,2\right) a^{\dagger}\left(1^{\prime}\right) a^{\dagger}\left(2^{\prime}\right) a(2) a(1) .
$$

Further, we will employ the transformation properties of the creation and annihilation operators with respect to $\Pi$. For example, in case of a massive particle with the mass $m$ and spin $j$ one considers that

$$
\begin{gathered}
U_{F}(\Lambda, b) a^{\dagger}(p, \mu) U_{F}^{-1}(\Lambda, b)=e^{i \Lambda p b} D_{\mu^{\prime} \mu}^{(j)}(W(\Lambda, p)) a^{\dagger}\left(\Lambda p, \mu^{\prime}\right), \\
\forall \Lambda \in L_{+} \text {and arbitrary spacetime shifts } \mathrm{b}=\left(\mathrm{b}^{0}, \mathbf{b}\right)
\end{gathered}
$$

with $D$-function whose argument is the Wigner rotation $W(\Lambda, p), L_{+}$the homogeneous (proper) orthochronous Lorentz group. The correspondence $(\Lambda, b) \rightarrow U_{F}(\Lambda, b)$ between elements $(\Lambda, b) \in \Pi$ and unitary transformations $U_{F}(\Lambda, b)$ realizes an irreducible representation of $\Pi$ on the Hilbert space $\mathcal{H}$ (to be definite) of meson-nucleon states. In this context, it is convenient to employ the operators $a(p, \mu)=a(\mathbf{p}, \mu) \sqrt{p_{0}}$ that meet the covariant commutation relations

$$
\begin{aligned}
{\left[a\left(p^{\prime}, \mu^{\prime}\right), a^{\dagger}(p, \mu)\right]_{ \pm} } & =p_{0} \delta\left(\mathbf{p}-\mathbf{p}^{\prime}\right) \delta_{\mu^{\prime} \mu} \\
{\left[a\left(p^{\prime}, \mu^{\prime}\right), a(p, \mu)\right]_{ \pm} } & =\left[a^{\dagger}\left(p^{\prime}, \mu^{\prime}\right), a^{\dagger}(p, \mu)\right]_{ \pm}=0 .
\end{aligned}
$$

Here $p_{0}=\sqrt{\mathbf{p}^{2}+m^{2}}$ is the fourth component of the 4-momentum $p=\left(p_{0}, \mathbf{p}\right)$.

\section{A possible way for constructing generators of the Poincaré group}

Let us recall that within the Lagrangian formalism the 4-vector $P^{\mu}=(H, \mathbf{P})$ for any local field model, where requirements of relativistic symmetry are manifestly provided at the beginning, is determined by the Nöther integrals

$$
P^{v}=\int \mathcal{T}^{0 v}(\mathbf{x}) d \mathbf{x} \quad(v=0,1,2,3),
$$


where $\mathcal{T}^{0 v}(\mathbf{x})$ are the components of the energy-momentum tensor density $\mathcal{T}^{\mu v}(x)$ at $t=0$.

Other Nöther integrals are expressed through the angular-momentum tensor density

$$
\mathcal{M}^{\beta[\mu v]}(x)=x^{\mu} \mathcal{T}^{\beta v}(x)-x^{v} \mathcal{T}^{\beta \mu}(x)+\Sigma^{\beta[\mu v]}(x),
$$

that contains, in general, so-called polarization part $\Sigma^{\beta[\mu v] 3}$ associated with spin degrees of freedom. Namely, the six independent integrals

$$
M^{\mu \nu}=\left.\int \mathcal{M}^{0[\mu \nu]}(x) d \mathbf{x}\right|_{t=0}
$$

are considered as the generators of space rotations

$$
J^{i}=\varepsilon_{i k l} M^{k l} \quad(i, k, l=1,2,3)
$$

and the boosts

$$
N^{k} \equiv M^{0 k}=-\int x^{k} \mathcal{T}^{00}(\mathbf{x}) d \mathbf{x}+\int \Sigma^{0[0 k]}(\mathbf{x}) d \mathbf{x},(k=1,2,3) .
$$

The reminder is not accidental as far as we strive to go out beyond the traditional QFT with local Lagrangian densities via special regularization of interactions in a total initial Hamiltonian.

\subsection{The Belinfante ansatz. Application to interacting pion and nucleon fields}

Regarding an illustration of these general relations let us write, the Lagrangian density

$$
\begin{gathered}
\mathcal{L}_{S C H}(x)=\frac{1}{2} \bar{\psi}_{H}(x)\left(i \gamma^{\mu} \vec{\partial}_{\mu}-m_{0}\right) \psi_{H}(x)+\frac{1}{2} \bar{\psi}_{H}(x)\left(-i \gamma^{\mu} \overleftarrow{\partial}_{\mu}-m_{0}\right) \psi_{H}(x) \\
+\frac{1}{2}\left[\partial_{\mu} \varphi_{H}(x) \partial^{\mu} \varphi_{H}(x)-\mu_{0}^{2} \varphi_{H}^{2}(x)\right]-i g_{0} \bar{\psi}_{H}(x) \gamma_{5} \psi_{H}(x) \varphi_{H}(x),
\end{gathered}
$$

for interacting pion $\phi$ and nucleon $\psi$ fields with the PS coupling (see, e.g.,(Schweber, 1961)). Then, one has (omitting argument $x$ ): i) energy-momentum tensor density

$$
\begin{aligned}
\mathcal{T}_{S C H}^{\mu \nu}=\frac{\partial \mathcal{L}_{S C H}}{\partial \bar{\psi}_{H} \mu} \bar{\psi}_{H}^{v} & +\frac{\partial \mathcal{L}_{S C H}}{\partial \psi_{H \mu}} \psi_{H}^{v}+\frac{\partial \mathcal{L}_{S C H}}{\partial \varphi_{H \mu}} \varphi_{H}^{v}-g^{\mu v} \mathcal{L}_{S C H} \\
& \equiv \mathcal{T}_{N}^{\mu v}+\mathcal{T}_{\pi}^{\mu \nu}+\mathcal{T}_{I}^{\mu \nu}
\end{aligned}
$$

where

$$
\begin{gathered}
\mathcal{T}_{N}^{\mu v}=\frac{i}{2} \bar{\psi}_{H} \gamma^{\mu} \partial^{v} \psi_{H}-\frac{i}{2} \gamma^{\mu} \psi_{H} \partial^{v} \bar{\psi}_{H}-g^{\mu v} \mathcal{L}_{N}, \\
\mathcal{T}_{\pi}^{\mu v}=\partial^{\mu} \varphi_{H} \partial^{v} \varphi_{H}-g^{\mu v} \mathcal{L}_{\pi}, \\
\mathcal{T}_{I}^{\mu \nu}=i g_{0} g^{\mu v} \bar{\psi}_{H} \gamma_{5} \psi_{H} \varphi_{H},
\end{gathered}
$$

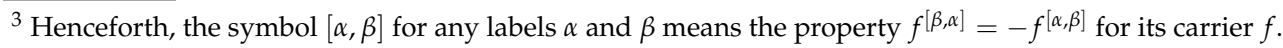


and ii) polarization contribution

$$
\Sigma_{S C H}^{\beta[\mu v]}=\frac{1}{2} i \bar{\psi}_{H}\left\{\gamma^{\beta} \Sigma^{\mu \nu}+\Sigma^{\mu v} \gamma^{\beta}\right\} \psi_{H},
$$

where

$$
\Sigma^{\mu \nu}=\frac{i}{4}\left[\gamma^{\mu}, \gamma^{v}\right]
$$

In formulae (21)-(25) unlike operators $O(x)$ in the D picture, we have operators

$$
O_{H}(x)=e^{i H t} O(\mathbf{x}) e^{-i H t},
$$

in the Heisenberg picture. We prefer to employ the definitions:

$$
\left\{\gamma^{\mu}, \gamma^{\nu}\right\}=2 g^{\mu \nu}, \gamma_{\mu}^{\dagger}=\gamma_{0} \gamma_{\mu} \gamma_{0},\left\{\gamma_{\mu}, \gamma_{5}\right\}=0, \gamma_{5}^{\dagger}=\gamma_{0} \gamma_{5} \gamma_{0}=-\gamma_{5} .
$$

The corresponding Hamiltonian density is given by

$$
H_{S C H}(\mathbf{x})=\mathcal{T}_{S C H}^{00}(\mathbf{x})=H_{f e r m}^{0}(\mathbf{x})+H_{\pi}^{0}(\mathbf{x})+V_{p s}^{0}(\mathbf{x}),
$$

where

$$
\begin{gathered}
H_{\text {ferm }}^{0}(\mathbf{x})=\frac{1}{2} \bar{\psi}(\mathbf{x})\left[-i \vec{\gamma} \vec{\partial}+m_{0}\right] \psi(\mathbf{x})+\frac{1}{2} \bar{\psi}(\mathbf{x})\left[+i \overleftarrow{\gamma} \overleftarrow{\partial}+m_{0}\right] \psi(\mathbf{x}), \\
H_{\pi}^{0}(\mathbf{x})=\frac{1}{2}\left[\pi^{2}(\mathbf{x})+\nabla \varphi(\mathbf{x}) \nabla \varphi(\mathbf{x})+\mu_{0}^{2} \varphi^{2}(\mathbf{x})\right], \\
V_{p s}^{0}(\mathbf{x})=i g_{0} \bar{\psi}(\mathbf{x}) \gamma_{5} \psi(\mathbf{x}) \varphi(\mathbf{x}),
\end{gathered}
$$

where, as usually, $\pi(\mathbf{x})$ denotes the canonical conjugate variable for the pion field. One should note that the second integral in the r.h.s. of Eq. (20) does not contribute to the model boost since operator (26) with $\beta=\mu=0$ and $v=k$ is identically equal zero. Thus we arrive to the relation

$$
\mathbf{N}_{S C H}=-\int \mathbf{x} \mathcal{T}_{S C H}^{00}(\mathbf{x}) d \mathbf{x}=-\int \mathbf{x} H_{S C H}(\mathbf{x}) d \mathbf{x},
$$

that exemplifies the so-called Belinfante ansatz:

$$
\mathbf{N}=-\int \mathbf{x} H(\mathbf{x}) d \mathbf{x}
$$

which, as it has first been shown in (Belinfante, 1940), holds for any local field model with a symmetrized density $\mathcal{T}^{\mu v}(x)=\mathcal{T}^{v \mu}(x)$. Such a representation helps (Shebeko \& Shirokov, 2001) to get simultaneously a sparse structure for the Hamiltonian and the generators of Lorentz boosts in the CPR ${ }^{4}$. We shall come back to this point later.

Further, the Hamiltonian density can be represented as

$$
H_{S C H}(\mathbf{x})=H_{F}(\mathbf{x})+H_{I}(\mathbf{x})
$$

\footnotetext{
$\overline{4}$ The relation (32) also has turned out to be useful when formulating a local analog of the Siegert theorem in the covariant description of electromagnetic interactions with nuclei (Shebeko, 1990).
} 
with the free part

$$
H_{F}(\mathbf{x})=H_{\pi}(\mathbf{x})+H_{\text {ferm }}(\mathbf{x})
$$

and the interaction density

$$
H_{I}(\mathbf{x})=V_{p s}(\mathbf{x})+H_{r e n}(\mathbf{x}), V_{p s}(\mathbf{x})=i g \bar{\psi}(\mathbf{x}) \gamma_{5} \psi(\mathbf{x}) \varphi(\mathbf{x}),
$$

where we have introduced the mass and vertex counterterms:

$$
\begin{gathered}
H_{r e n}(\mathbf{x})=M_{r e n}^{m e s}(\mathbf{x})+M_{r e n}^{f e r m}(\mathbf{x})+H_{r e n}^{i n t}(\mathbf{x}), \\
M_{r e n}^{\text {mes }}(\mathbf{x})=\frac{1}{2}\left(\mu_{0}^{2}-\mu_{\pi}^{2}\right) \varphi^{2}(\mathbf{x}), \\
M_{r e n}^{f e r m}(\mathbf{x})=\left(m_{0}-m\right) \bar{\psi}(\mathbf{x}) \psi(\mathbf{x})
\end{gathered}
$$

and

$$
H_{r e n}^{i n t}(\mathbf{x})=i\left(g_{0}-g\right) \bar{\psi}(\mathbf{x}) \gamma_{5} \psi(\mathbf{x}) \varphi(\mathbf{x}) .
$$

One should note that the densities in Eqs. (34)-(35) are obtained from Eqs. (28)-(29) replacing the bare values $m_{0}, \mu_{0}$ and $g_{0}$, respectively, by the "physical" values $m, \mu_{\pi}$ and $g$. Such a transition can be done via the mass-changing Bogoliubov-type transformations (details in (Korda et al., 2007)). In particular, the fields involved can be expressed through the set $\alpha=a^{\dagger}(a), b^{\dagger}(b), d^{\dagger}(d)$ of the creation (destruction) operators for the bare pions and nucleons with the physical masses,

$$
\begin{gathered}
\varphi(\mathbf{x})=(2 \pi)^{-3 / 2} \int\left(2 \omega_{\mathbf{k}}\right)^{-1 / 2}\left[a(\mathbf{k})+a^{\dagger}(-\mathbf{k})\right] \exp (i \mathbf{k x}) d \mathbf{k}, \\
\pi(\mathbf{x})=-i(2 \pi)^{-3 / 2} \int\left(\omega_{\mathbf{k}} / 2\right)^{1 / 2}\left[a(\mathbf{k})-a^{\dagger}(-\mathbf{k})\right] \exp (i \mathbf{k} \mathbf{x}) d \mathbf{k}, \\
\psi(\mathbf{x})=(2 \pi)^{-3 / 2} \int(m / E \mathbf{p})^{1 / 2} \sum_{\mu}[u(\mathbf{p} \mu) b(\mathbf{p} \mu) \\
\left.+v(-\mathbf{p} \mu) d^{\dagger}(-\mathbf{p} \mu)\right] \exp (i \mathbf{p} \mathbf{x}) d \mathbf{p} .
\end{gathered}
$$

Substituting (33) into (31), we find

$$
\mathbf{N}=\mathbf{N}_{F}+\mathbf{N}_{I}
$$

with

$$
\mathbf{N}_{F}=\mathbf{N}_{f e r m}+\mathbf{N}_{\pi}=-\int \mathbf{x} H_{f e r m}(\mathbf{x}) d \mathbf{x}-\int \mathbf{x} H_{\pi}(\mathbf{x}) d \mathbf{x}
$$

and

$$
\mathbf{N}_{I}=-\int \mathbf{x} H_{I}(\mathbf{x}) d \mathbf{x}
$$

Now, taking into account the transformation properties of the fermion field $\psi(x)$ and the pion field $\varphi(x)$ with respect to $\Pi$, it is readily seen that in the D picture density (33) is a scalar, i.e.,

$$
U_{F}(\Lambda, b) H_{S C H}(x) U_{F}^{-1}(\Lambda, b)=H_{S C H}(\Lambda x+b),
$$

so

$$
U_{F}(\Lambda, b) H_{I}(x) U_{F}^{-1}(\Lambda, b)=H_{I}(\Lambda x+b) .
$$


It is well known (see, e.g., Sect. 5.1 in (Weinberg, 1995)) that for a large class of theories the property (41) with the corresponding interaction densities $H_{I}(x)$, being supplemented by the condition

$$
\left[H_{I}\left(x^{\prime}\right), H_{I}(x)\right]=0 \text { for }\left(x^{\prime}-x\right)^{2} \leq 0,
$$

plays a crucial role for covariant calculations of the $S$-matrix.

\subsection{An algebraic approach within the Hamiltonian formalism}

After these preliminaries, let us consider field models with the decomposition

$$
H_{I}=H_{s c}+H_{n s c} \equiv \int H_{s c}(\mathbf{x}) d \mathbf{x}+\int H_{n s c}(\mathbf{x}) d \mathbf{x} .
$$

It means that only the density in the first integral has the property (41), i.e.,

$$
U_{F}(\Lambda, b) H_{s c}(x) U_{F}^{-1}(\Lambda, b)=H_{s c}(\Lambda x+b) .
$$

It is the case, where the pseudoscalar $(\pi$ and $\eta)$, vector $(\rho$ and $\omega)$ and scalar $(\delta$ and $\sigma)$ meson (boson) fields interact with the $1 / 2 \operatorname{spin}(N$ and $\bar{N})$ fermion ones via the Yukawa-type couplings $V=\sum_{b} V_{b}=V_{s}+V_{p s}+V_{\mathrm{v}}$ in

$$
H_{I}=V+\text { mass and vertex counterterms }
$$

with

$$
\begin{gathered}
V_{s}=g_{s} \int d \vec{x} \bar{\psi}(\vec{x}) \psi(\vec{x}) \varphi_{s}(\vec{x}), \\
V_{p s}=i g_{p s} \int d \vec{x} \bar{\psi}(\vec{x}) \gamma_{5} \psi(\vec{x}) \varphi_{p s}(\vec{x})
\end{gathered}
$$

and

$$
\begin{aligned}
V_{\mathrm{v}}=\int d \vec{x} & \left\{g_{\mathrm{v}} \bar{\psi}(\vec{x}) \gamma_{\mu} \psi(\vec{x}) \varphi_{\mathrm{v}}^{\mu}(\vec{x})+\frac{f_{\mathrm{v}}}{4 m} \bar{\psi}(\vec{x}) \sigma_{\mu \nu} \psi(\vec{x}) \varphi_{\mathrm{v}}^{\mu v}(\vec{x})\right\} \\
& +\int d \vec{x}\left\{\frac{g_{\mathrm{v}}^{2}}{2 m_{\mathrm{v}}^{2}} \bar{\psi}(\vec{x}) \gamma_{0} \psi(\vec{x}) \bar{\psi}(\vec{x}) \gamma_{0} \psi(\vec{x})+\frac{f_{\mathrm{v}}^{2}}{4 m^{2}} \bar{\psi}(\vec{x}) \sigma_{0 i} \psi(\vec{x}) \bar{\psi}(\vec{x}) \sigma_{0 i} \psi(\vec{x})\right\},
\end{aligned}
$$

where $\varphi_{\mathrm{v}}^{\mu \nu}(\vec{x})=\partial^{\mu} \varphi_{\mathrm{v}}^{v}(\vec{x})-\partial^{v} \varphi_{\mathrm{v}}^{\mu}(\vec{x})$ is the tensor of the vector fields involved (details in (Dubovyk \& Shebeko, 2010)).

In the context we would like to remind that in "...theories with derivative couplings or spins $j \geq 1$, it is not enough to take Hamiltonian as the integral over space of a scalar interaction density; we also need to add non-scalar terms to the interaction density to compensate non-covariant terms in the propagators" (quoted from Chapter VII in (Weinberg, 1995)).

Then, taking into account that the first relation (11) is equivalent to the equality

$$
\left[\mathbf{N}_{F}, H_{I}\right]=\left[H, \mathbf{N}_{I}\right]
$$

we will evaluate its l.h.s.. In this connection, let us regard the operator

$$
H_{s c}(t)=\int H_{s c}(x) d \mathbf{x}
$$


and its similarity transformation

$$
e^{i \vec{\beta} \mathbf{N}_{F}} H_{S C}(t) e^{-i \vec{\beta} \mathbf{N}_{F}}=\int H_{S C}(L(\vec{\beta}) x) d \mathbf{x},
$$

where $L(\vec{\beta})$ is any Lorentz boost with the parameters $\vec{\beta}=\left(\beta^{1}, \beta^{2}, \beta^{3}\right)$.

From (51) it follows that

$$
i e^{i \beta^{1} N_{F}^{1}}\left[N_{F}^{1}, H_{s c}(t)\right] e^{-i \beta^{1} N_{F}^{1}}=\frac{\partial}{\partial \beta^{1}} \int H_{s c}\left(L\left(\beta^{1}\right) x\right) d \mathbf{x},
$$

whence, for instance,

$$
\begin{aligned}
i\left[N_{F}^{1}, H_{s c}(t)\right] & =\lim _{\beta^{1} \rightarrow 0} \frac{\partial}{\partial \beta^{1}} \int H_{s c}\left(t-\beta^{1} x^{1}, x^{1}-\beta^{1} t, x^{2}, x^{3}\right) d \mathbf{x} \\
& =-\int\left(t \frac{\partial}{\partial x^{1}} H_{s c}(x)+x^{1} \frac{\partial}{\partial t} H_{s c}(x)\right) d \mathbf{x},
\end{aligned}
$$

since for the infinitesimal boost

$$
L(\vec{\beta}) x=(t-\vec{\beta} \mathbf{x}, \mathbf{x}-\vec{\beta} t) .
$$

In turn, from (53) we get

$$
\left[N_{F}^{1}, H_{s c}\right]=i \lim _{t \rightarrow 0} \int\left(-i t\left[P^{1}, H_{s c}(x)\right]+i x^{1}\left[H_{F}, H_{s c}(x)\right]\right) d \mathbf{x}
$$

so

$$
\left[\mathbf{N}_{F}, H_{s c}\right]=-\int \mathbf{x}\left[H_{F}, H_{s c}(\mathbf{x})\right] d \mathbf{x} .
$$

By using Eq. (54) equality (49) can be written as

$$
-\int \mathbf{x}\left[H_{F}, H_{s c}(\mathbf{x})\right] d \mathbf{x}=\left[H_{F}, \mathbf{N}_{I}\right]+\left[H_{I}, \mathbf{N}_{I}\right]+\left[H_{n s \mathcal{C}}, \mathbf{N}_{F}\right] .
$$

Evidently, this equation is fulfilled if we put

$$
\mathbf{N}_{I}=\mathbf{N}_{B} \equiv-\int \mathbf{x} H_{s c}(\mathbf{x}) d \mathbf{x}
$$

and

or

$$
\left[H_{s c}, \mathbf{N}_{I}\right]=-\int \mathbf{x} d \mathbf{x} \int d \mathbf{x}^{\prime}\left[H_{s c}\left(\mathbf{x}^{\prime}\right), H_{s c}(\mathbf{x})\right]=\left[\mathbf{N}_{F}+\mathbf{N}_{I}, H_{n s c}\right]
$$

$$
\begin{aligned}
& \int d \mathbf{x} \int d \mathbf{x}^{\prime}\left(\mathbf{x}^{\prime}-\mathbf{x}\right)\left[H_{S C}\left(\mathbf{x}^{\prime}\right), H_{S C}(\mathbf{x})\right] \\
= & \int \mathbf{x} d \mathbf{x} \int d \mathbf{x}^{\prime}\left[H_{n s C}\left(\mathbf{x}^{\prime}\right), H_{F}(\mathbf{x})+H_{S C}(\mathbf{x})\right] .
\end{aligned}
$$

In a model with $H_{n s c}=0$ the latter reduces to

$$
\int e^{-i \mathbf{P X}} \mathbf{I} e^{i \mathbf{P X}} d \mathbf{X}=0
$$


where

$$
\mathbf{I}=\frac{1}{2} \int \mathbf{r} d \mathbf{r}\left[H_{S C}\left(\frac{1}{2} \mathbf{r}\right), H_{S C}\left(-\frac{1}{2} \mathbf{r}\right)\right]
$$

By running again the way from Eq. (49) to Eqs. (59)-(60) we see that the nonlinear commutation (11)

$$
[H, \mathbf{N}]=i \mathbf{P}
$$

will take place once along with the Belinfante-type relation (56) the interaction density meets the condition

$$
\int \mathbf{r} d \mathbf{r}\left[H_{s c}\left(\frac{1}{2} \mathbf{r}\right), H_{s c}\left(-\frac{1}{2} \mathbf{r}\right)\right]=0 .
$$

One should note that we have arrived to Eq. (56) being inside the Poincarè algebra itself without addressing the Nöther integrals, these stepping stones of the Lagrangian formalism. In the context, we would like to stress that the condition (61) is weaker compared to the constraint

$$
\left[H_{s c}\left(\frac{1}{2} \mathbf{r}\right), H_{s c}\left(-\frac{1}{2} \mathbf{r}\right)\right]=0
$$

imposed for all $\mathbf{r}$ excepting, may be, the point $\mathbf{r}=0$. But we recall it as a special case of the microcausality requirement that is realized in local field models. Beyond such models, as shown in Appendix B of (Shebeko \& Frolov , 2011), Eqs. (56) and (49) may be incompatible. It makes us seek an alternative to assumption (56) in our attempts to meet Eq. (55).

At this point, we put $\mathbf{N}_{I}=\mathbf{N}_{B}+\mathbf{D}$ to get the relationship

$$
\left[H_{F}, \mathbf{D}\right]=\left[\mathbf{N}_{B}+\mathbf{D}, H_{s c}\right]+\left[\mathbf{N}_{F}+\mathbf{N}_{B}+\mathbf{D}, H_{n s c}\right],
$$

that replaces the commutator $[H, \mathbf{N}]=i \mathbf{P}$ and determines the displacement $\mathbf{D}$.

Further, assuming that the scalar density $H_{s c}(\mathbf{x})$ is of the first order in coupling constants involved and putting

$$
H_{n s c}(\mathbf{x})=\sum_{p=2}^{\infty} H_{n s c}^{(p)}(\mathbf{x}),
$$

we will search the operator $\mathbf{D}$ in the form

$$
\mathbf{D}=\sum_{p=2}^{\infty} \mathbf{D}^{(p)},
$$

i.e., as a perturbation expansion in powers of the interaction $H_{s c}$. Here the label $(p)$ denotes the pth order in these constants. By the way, one should keep in mind that the terms in the r.h.s. of Eq. (64) are usually associated with perturbation series for mass and vertex counterterms. Evidently, their incorporation may affect the corresponding higher-order contributions with $p \geq 2$ to the boost. Therefore, to comprise different situations of practical interest let us consider field models in which $H_{n s c}(\mathbf{x})=V_{n s c}(\mathbf{x})+V_{\text {ren }}(\mathbf{x})$ with a nonscalar interaction $V_{n s c}=\int V_{n s c}(\mathbf{x}) d \mathbf{x}$ and some "renormalization" contribution $V_{r e n}=\int V_{\text {ren }}(\mathbf{x}) d \mathbf{x}$. The latter may be scalar or not. Of course, such a division of $H_{n s c}(\mathbf{x})$ can be done at the beginning in Eq. (43). But the scheme presented here seems to us more flexible. 
By substituting the expansions (64) and (65) into Eq. (63) we get the chain of relations

$$
\begin{gathered}
{\left[H_{F}, \mathbf{D}^{(2)}\right]=\left[\mathbf{N}_{F}, H_{n s c}^{(2)}\right]+\left[\mathbf{N}_{B}, H_{s c}\right],} \\
{\left[H_{F}, \mathbf{D}^{(3)}\right]=\left[\mathbf{N}_{F}, H_{n s c}^{(3)}\right]+\left[\mathbf{D}^{(2)}, H_{s c}\right]+\left[\mathbf{N}_{B}, H_{n s c}^{(2)}\right],} \\
{\left[H_{F}, \mathbf{D}^{(p)}\right]=\left[\mathbf{N}_{F}, H_{n s c}^{(p)}\right]+\left[\mathbf{N}_{B}, H_{n s c}^{(p-1)}\right]+\left[\mathbf{D}^{(p-1)}, H_{s c}\right]+\left[\mathbf{D}, H_{n s c}\right]{ }^{(p)},} \\
(p=4,5, \ldots)
\end{gathered}
$$

for a recursive finding of the operators $\mathbf{D}^{(p)}(p=2,3, \ldots)$.

Further, after such substitutions into the commutators

$$
\left[P_{k}, N_{j}\right]=i \delta_{k j} H, \quad\left[J_{k}, N_{j}\right]=i \varepsilon_{k j l} N_{l}, \quad\left[N_{k}, N_{j}\right]=-i \varepsilon_{k j l} J_{l}
$$

we deduce, respectively, the following relations:

$$
\left[P_{k}, D_{j}^{(p)}\right]=i \delta_{k j} H_{n s c}^{(p)} \quad(p=2,3, \ldots)
$$

from

$$
\begin{gathered}
{\left[P_{k}, D_{j}\right]=i \delta_{k j} H_{n s c},} \\
{\left[J_{k}, D_{j}^{(p)}\right]=i \varepsilon_{k j l} D_{l}^{(p)}}
\end{gathered}
$$

from

$$
\left[J_{k}, D_{j}\right]=i \varepsilon_{k j l} D_{l}
$$

and

$$
\left[N_{F k}, N_{B j}\right]+\left[N_{B k}, N_{F j}\right]=0,
$$

The remaining Poincaré commutations are fulfilled once one deals with any rotationally and translationally invariant theory.

Now, keeping in mind an elegant method by Chandler (Chandler, 2003), we invoke on the property (see (Friedrichs, 1953)) of a formal solution $Y$ of the equation

$$
\left[H_{F}, Y\right]=X
$$

to be any linear functional $F(X)$ of a given operator $X \neq 0$. In other words, it means that

$$
\left[H_{F}, F(X)\right]=X
$$

with $F\left(\lambda_{1} X_{1}+\lambda_{2} X_{2}\right)=\lambda_{1} F\left(X_{1}\right)+\lambda_{2} F\left(X_{2}\right)$, where $\lambda_{1}$ and $\lambda_{2}$ are arbitrary c-numbers. In addition, one can see that

$$
\left[H_{F}, F(X)\right]=F\left(\left[H_{F}, X\right]\right) .
$$

Moreover, it turns out that

$$
\begin{gathered}
{[\mathbf{P}, F(X)]=F([\mathbf{P}, X])} \\
{[\mathbf{J}, F(X)]=F([\mathbf{J}, X])} \\
{\left[\mathbf{N}_{F}, F(X)\right]=F\left(\left[\mathbf{N}_{F}, X\right]\right)+i F(F([\mathbf{P}, X])) .}
\end{gathered}
$$


In order to prove the relations let us employ the Jacobi identity

$$
[A,[B, C]]+[C,[A, B]]+[B,[C, A]]=0
$$

and write

$$
\left[\mathcal{O},\left[H_{F}, F(X)\right]\right]=-\left[F(X),\left[\mathcal{O}, H_{F}\right]\right]+\left[H_{F},[\mathcal{O}, F(X)]\right]
$$

with some operator $\mathcal{O}$. Then

$$
[\mathcal{O}, F(X)]=F([\mathcal{O}, X])+F\left(\left[F(X),\left[\mathcal{O}, H_{F}\right]\right]\right) .
$$

Of course, to be more constructive one needs to have a definite realization of the functional $F(X)$. In this connection, we will use the representation

$$
Y=-i \lim _{\eta \rightarrow 0+} \int_{0}^{\infty} X(t) e^{-\eta t} d t
$$

of the operator $Y$ that enters the equation (74). The existence proof for such a solution is sufficiently delicate (see discussion in Appendix A of Ref. (Shebeko \& Shirokov, 2001)).

\subsection{Application to a nonlocal field model}

We will show how the method proposed works in combination with introducing certain cutoff (vertex) functions that makes an initial local model be nonlocal. In spite of our consideration may be extended to more realistic models its main idea becomes transparent for a simple system of "scalar nucleons" (more precisely, charged spinless bosons) and neutral scalar bosons with the interaction density $H_{I}(\mathbf{x})=V_{l o c}(\mathbf{x})+V_{\text {ren }}(\mathbf{x})$ (cf. (Glöckle \& Müller, 1981; Shirokov, 2002)):

$$
V_{l o c}(\mathbf{x})=g \varphi_{s}(\mathbf{x}): \psi_{b}^{\dagger}(\mathbf{x}) \psi_{b}(\mathbf{x}):
$$

and

$$
V_{r e n}(\mathbf{x})=\delta \mu_{s}: \varphi_{s}^{2}(\mathbf{x}):+\delta \mu_{b}: \psi_{b}^{\dagger}(\mathbf{x}) \psi_{b}(\mathbf{x}):
$$

with the mass shifts $\delta \mu_{s}=\frac{1}{2}\left(\mu_{0 s}^{2}-\mu_{s}^{2}\right), \delta \mu_{b}=\frac{1}{2}\left(\mu_{0 b}^{2}-\mu_{b}^{2}\right)$. In order to regard a nonlocal extension of this local model let us substitute the expansions

$\varphi_{s}(\mathbf{x})=\left[2(2 \pi)^{3}\right]^{-1 / 2} \int \frac{d \mathbf{k}}{\omega_{\mathbf{k}}}\left[a(k)+a^{\dagger}\left(k_{-}\right)\right] e^{i \mathbf{k x}}, \quad \psi_{b}(\mathbf{x})=\left[2(2 \pi)^{3}\right]^{-1 / 2} \int \frac{d \mathbf{p}}{E_{\mathbf{p}}}\left[b(p)+d^{\dagger}\left(p_{-}\right)\right] e^{i \mathbf{p x}}$

into Eqs. (83) and (84) to get

$$
\begin{gathered}
V_{l o c}(\mathbf{x})=\frac{g}{2\left[2(2 \pi)^{3}\right]^{1 / 2}} \int \frac{d \mathbf{p}^{\prime}}{E_{\mathbf{p}^{\prime}}} \int \frac{d \mathbf{p}}{E_{\mathbf{p}}} \int \frac{d \mathbf{k}}{\omega_{\mathbf{k}}} e^{-i \mathbf{p}^{\prime} \mathbf{x}+i \mathbf{p} \mathbf{x}+i \mathbf{k x}} \\
\times a(k):\left[b^{\dagger}\left(p^{\prime}\right) b(p)+b^{\dagger}\left(p^{\prime}\right) d^{\dagger}\left(p_{-}\right)+d\left(p_{-}^{\prime}\right) b(p)+d\left(p_{-}^{\prime}\right) d^{\dagger}\left(p_{-}\right)\right]:+ \text {H.c. }
\end{gathered}
$$

and $V_{r e n}(\mathbf{x})=\delta \mu_{s}(\mathbf{x})+\delta \mu_{b}(\mathbf{x})$ with

$$
\delta \mu_{S}(\mathbf{x})=\frac{\delta \mu_{S}}{2(2 \pi)^{3}} \int \frac{d \mathbf{k}^{\prime}}{\omega_{\mathbf{k}^{\prime}}} \int \frac{d \mathbf{k}}{\omega_{\mathbf{k}}}:\left[a\left(k^{\prime}\right)+a^{\dagger}\left(k_{-}^{\prime}\right)\right] e^{i \mathbf{k}^{\prime} \mathbf{x}+i \mathbf{k} \mathbf{x}}\left[a(k)+a^{\dagger}\left(k_{-}\right)\right]:,
$$




$$
\delta \mu_{b}(\mathbf{x})=\frac{\delta \mu_{b}}{2(2 \pi)^{3}} \int \frac{d \mathbf{p}^{\prime}}{E_{\mathbf{p}^{\prime}}} \int \frac{d \mathbf{p}}{E_{\mathbf{p}}}:\left[b^{\dagger}\left(p^{\prime}\right)+d\left(p_{-}^{\prime}\right)\right] e^{-i \mathbf{p}^{\prime} \mathbf{x}+i \mathbf{p x}}\left[b(p)+d^{\dagger}\left(p_{-}\right)\right]: .
$$

It is implied that the operators $a\left(a^{\dagger}\right), b\left(b^{\dagger}\right)$ and $d\left(d^{\dagger}\right)$ meet the commutation relations

$$
\begin{gathered}
{\left[a(k), a^{\dagger}\left(k^{\prime}\right)\right]=k_{0} \delta\left(\mathbf{k}-\mathbf{k}^{\prime}\right),} \\
{\left[b(p), b^{\dagger}\left(p^{\prime}\right)\right]=\left[d(p), d^{\dagger}\left(p^{\prime}\right)\right]=p_{0} \delta\left(\mathbf{p}-\mathbf{p}^{\prime}\right)}
\end{gathered}
$$

with all the remaining ones being zero.

The interaction operator itself $H_{I}=\int H_{I}(\mathbf{x}) d \mathbf{x}=V_{l o c}+V_{\text {ren }}$ with

$$
\begin{gathered}
V_{l o c}=\int V_{\text {nloc }}(\mathbf{x}) d \mathbf{x}=\frac{g}{2\left[2(2 \pi)^{3}\right]^{1 / 2}} \int \frac{d \mathbf{p}^{\prime}}{E_{\mathbf{p}^{\prime}}} \int \frac{d \mathbf{p}}{E_{\mathbf{p}}} \int \frac{d \mathbf{k}}{\omega_{\mathbf{k}}} \delta\left(\mathbf{p}^{\prime}-\mathbf{p}-\mathbf{k}\right) \\
\times a(k):\left[b^{\dagger}\left(p^{\prime}\right) b(p)+b^{\dagger}\left(p^{\prime}\right) d^{\dagger}\left(p_{-}\right)+d\left(p_{-}^{\prime}\right) b(p)+d\left(p_{-}^{\prime}\right) d^{\dagger}\left(p_{-}\right)\right]:+H . c ., \\
V_{r e n}=\int\left[\delta \mu_{s}(\mathbf{x})+\delta \mu_{b}(\mathbf{x})\right] d \mathbf{x} .
\end{gathered}
$$

Let us consider its nonlocal extension

$$
H_{I}=V_{\text {nloc }}+M_{s}+M_{b}
$$

where in accordance with the representation (3) we introduce the following normally-ordered structures:

$$
\begin{gathered}
V_{\text {nloc }}=\int V_{\text {nloc }}(\mathbf{x}) d \mathbf{x}=\int \frac{d \mathbf{p}^{\prime}}{E_{\mathbf{p}^{\prime}}} \int \frac{d \mathbf{p}}{E_{\mathbf{p}}} \int \frac{d \mathbf{k}}{\omega_{\mathbf{k}}} \\
\times\left\{\delta\left(\mathbf{p}^{\prime}-\mathbf{p}-\mathbf{k}\right) g_{11}\left(p^{\prime}, p, k\right) b^{\dagger}\left(p^{\prime}\right) b(p)+\delta\left(\mathbf{p}^{\prime}+\mathbf{p}-\mathbf{k}\right) g_{12}\left(p^{\prime}, p, k\right) b^{\dagger}\left(p^{\prime}\right) d^{\dagger}(p)\right. \\
+\delta\left(\mathbf{p}^{\prime}+\mathbf{p}+\mathbf{k}\right) g_{21}\left(p^{\prime}, p, k\right) d\left(p^{\prime}\right) b(p) \\
\left.+\delta\left(\mathbf{p}^{\prime}-\mathbf{p}-\mathbf{k}\right) g_{22}\left(p^{\prime}, p, k\right) d^{\dagger}\left(p^{\prime}\right) d(p)\right\} a(k)+\text { H.c. }
\end{gathered}
$$

Furthermore, the creation/destruction operators have the transformation properties like (14). For example,

$$
U_{F}(\Lambda) a(k) U_{F}^{-1}(\Lambda)=a(\Lambda k) .
$$

Therefore, in the Dirac picture

$$
U_{F}(\Lambda) V_{l o c}(x) U_{F}^{-1}(\Lambda)=V_{l o c}(\Lambda x),
$$

i.e., the interaction density $V_{l o c}(x)$ is a Lorentz scalar.

For our nonlocal model we will retain the property assuming that

$$
U_{F}(\Lambda) V_{n l o c}(x) U_{F}^{-1}(\Lambda)=V_{n l o c}(\Lambda x) .
$$

It is readily seen that this relation holds if the coefficients $g_{\varepsilon^{\prime} \varepsilon}$ meet the condition

$$
g_{\varepsilon^{\prime} \varepsilon}\left(\Lambda p^{\prime}, \Lambda p, \Lambda k\right)=g_{\varepsilon^{\prime} \varepsilon}\left(p^{\prime}, p, k\right) .
$$


On the mass shells with $p^{2}=p^{2}=\mu_{b}^{2}$ and $k^{2}=\mu_{s}^{2}$ the latter means that the functions $g_{\varepsilon^{\prime} \varepsilon}\left(p^{\prime}, p, k\right)$ can depend only upon the invariants $p^{\prime} p, p^{\prime} k$ and $p k$.

The transition from $V_{l o c}$ to $V_{n l o c}$ can be interpreted as an endeavor to regularize the theory. In the context, the introduction of some cutoff functions $g_{\varepsilon^{\prime} \varepsilon}$ in momentum space is aimed at removing ultraviolet divergences typical of local field models with interactions like expression (83).

An associated exploration carried out in (Shebeko \& Frolov ,2011) with covariant cutoffs

$$
g_{\varepsilon^{\prime} \mathcal{\varepsilon}}\left(p^{\prime}, p, k\right)=v_{\mathcal{\varepsilon}^{\prime} \varepsilon}\left(\left[k+(-1)^{\varepsilon^{\prime}} p^{\prime}-(-1)^{\varepsilon} p\right]\left[k-(-1)^{\varepsilon^{\prime}} p^{\prime}+(-1)^{\varepsilon} p\right]\right)
$$

has allowed us to evaluate the lowest-order correction $\mathbf{D}^{(2)}$ to the Belinfante operator and get the leading-order analytic expressions for the coefficients in the "mass renormalization" terms

$$
\begin{gathered}
M_{s}=\int \frac{d \mathbf{k}}{\omega_{\mathbf{k}}^{2}}\left\{m_{1}(k) a^{\dagger}(k) a(k)+m_{2}(k)\left[a^{\dagger}(k) a^{\dagger}\left(k_{-}\right)+a(k) a\left(k_{-}\right)\right]\right\}, \\
M_{b}=\int \frac{d \mathbf{p}}{E_{\mathbf{p}}^{2}}\left\{m_{11}(p) b^{\dagger}(p) b(p)+m_{12}(p) b^{\dagger}(p) d^{\dagger}\left(p_{-}\right)+m_{21}(p) b(p) d\left(p_{-}\right)+m_{22}(p) d^{\dagger}(p) d(p)\right\} .
\end{gathered}
$$

\section{The method of unitary clothing transformations in action}

As shown in (Shebeko \& Shirokov, 2001), the Belinfante ansatz turns out to be useful when constructing the Lorentz boosts in the CPR, viz., the generator $\mathbf{N} \equiv \mathbf{N}(\alpha)$, being a function of the primary operators $\{\alpha\}$ (such as $a^{\dagger}(a), b^{\dagger}(b)$ and $d^{\dagger}(d)$ for the examples regarded above) in the BPR, is expressed through the corresponding operators $\left\{\alpha_{c}\right\}$ for particle creation and annihilation in the CPR. The transition $\{\alpha\} \Longrightarrow\left\{\alpha_{\mathcal{c}}\right\}$ is implemented via the special unitary transformations $W(\alpha)=W\left(\alpha_{c}\right)$, viz.,

$$
\alpha=W\left(\alpha_{c}\right) \alpha_{c} W^{\dagger}\left(\alpha_{c}\right) .
$$

These transformations satisfy certain physical requirements:

i) The physical vacuum (the $H$ lowest eigenstate) must coincide with a new no-particle state $\Omega$, i.e., the state that obeys the equations

$$
\begin{gathered}
a_{c}(\vec{k})|\Omega\rangle=b_{c}(\vec{p}, \mu)|\Omega\rangle=d_{c}(\vec{p}, \mu)|\Omega\rangle=0, \forall \vec{k}, \vec{p}, \mu \\
\langle\Omega \mid \Omega\rangle=1 .
\end{gathered}
$$

ii) New one-particle states $|\vec{k}\rangle_{c} \equiv a_{c}^{\dagger}(\vec{k}) \Omega$ etc. are the $H$ eigenvectors as well.

$$
\begin{gathered}
K\left(\alpha_{c}\right)|\vec{k}\rangle_{c}=K_{F}\left(\alpha_{c}\right)|\vec{k}\rangle_{c}=\omega_{k}|\vec{k}\rangle_{c} \\
K_{I}\left(\alpha_{c}\right)|\vec{k}\rangle_{c}=0
\end{gathered}
$$

iii) The spectrum of indices that enumerate the new operators must be the same as that for the bare ones . 
iv) The new operators $\alpha_{\mathcal{c}}$ satisfy the same commutation rules as do their bare counterparts $\alpha$ that is provided via the link (101) with a unitary operator $W$ to be obtained as in (Shebeko \& Shirokov, 2001).

\subsection{The Hamiltonian and other generators of the Poincare group in the clothed-particle representation}

A key point of the clothing procedure exposed in (Shebeko \& Shirokov, 2001) is to remove the so-called bad terms from the Hamiltonian

$$
H \equiv H(\alpha)=H_{F}(\alpha)+H_{I}(\alpha)=W\left(\alpha_{c}\right) H\left(\alpha_{c}\right) W^{\dagger}\left(\alpha_{c}\right) \equiv K\left(\alpha_{c}\right),
$$

more exactly, from a primary interaction $V(\alpha)$ in $H_{I}(\alpha)=V(\alpha)+V_{\text {ren }}(\alpha)$. For example, these terms $b_{c}^{\dagger} b_{c} a_{c}^{\dagger}, b_{c}^{\dagger} d_{c}^{\dagger} a_{c}, b_{c}^{\dagger} d_{c}^{\dagger} a_{c}^{\dagger}, d_{c} d_{c}^{\dagger} a_{c}^{\dagger}$ enter $V\left(\alpha_{c}\right)$ determined by Eq. (90) after the replacement of the bare operators in it by the clothed ones. These terms should be removed together with their Hermitian conjugate counterterms! to retain the hermiticity of the similarity transformation (105). In general, such terms prevent the physical vacuum $|\Omega\rangle$ (the $H$ lowest eigenstate) and the one-clothed-particle states $|n\rangle_{c}=a_{c}^{\dagger}(n)|\Omega\rangle$ to be the $H$ eigenvectors for all $n$ included. Here creation operators $a_{c}^{\dagger}(n)$ are clothed counterparts of those operators $a^{\dagger}(n)$ that are contained in expansion (2). The bad terms occur every time when any normally ordered product

$$
a^{\dagger}\left(1^{\prime}\right) a^{\dagger}\left(2^{\prime}\right) \ldots a^{\dagger}\left(n_{C}^{\prime}\right) a\left(n_{A}\right) \ldots a(2) a(1)
$$

of the class [C.A] embodies, at least, one substructure which belongs to one of the classes $[k .0]$ $(k=1,2, \ldots)$ and $[k .1](k=0,1, \ldots)$.

Strictly speaking such a departure point should be specified and sometimes modified. Indeed, by trying to meet the requirements i) and ii) we, at first sight, leave out of consideration such undesirable terms in $V_{r e n}(\alpha)$. Nevertheless, it is not accidental since the renormalization contribution is canceled in the course of the procedure itself that is some attractive feature of the UCT method as a whole (see below). In addition, it has turned out (Dubovyk \& Shebeko, 2010) that the nonscalar contribution (the second integral in the r.h.s. of Eq. (48)) to the operator $V_{\mathrm{v}}(\alpha)$ is canceled too when eliminating bad terms only from its scalar part (in fact, the first integral in the r.h.s. of Eq. (48)). Keeping this in mind, when handling the division

$$
H_{I}(\alpha)=\int H_{I}(\mathbf{x}) d \mathbf{x}=H_{s c}(\alpha)+H_{n s c}(\alpha)
$$

we assume $H_{s c}(\alpha)=V_{b a d}(\alpha)+V_{\text {good }}(\alpha)$ to remove the bad part $V_{b a d}$ from the similarity transformation

$$
\begin{gathered}
K\left(\alpha_{c}\right)=W\left(\alpha_{c}\right)\left[H_{F}\left(\alpha_{c}\right)+H_{I}\left(\alpha_{c}\right)\right] W^{\dagger}\left(\alpha_{c}\right) \\
=W\left(\alpha_{c}\right)\left[H_{F}\left(\alpha_{c}\right)+V_{\text {bad }}\left(\alpha_{c}\right)+V_{\text {good }}\left(\alpha_{c}\right)+H_{n s c}\left(\alpha_{c}\right)\right] W^{\dagger}\left(\alpha_{c}\right) .
\end{gathered}
$$

Remind that term "good", as an antithesis of "bad", is applied here to those operators (e.g., of the class [k.2] with $k \geq 2$ ) which destroy both the no-clothed-particle state $\Omega$ and the one-clothed-particle states. 
For unitary transformation (UCT) $W=\exp R$ with $R=-R^{\dagger}$ it is implied that we will eliminate the bad terms $V_{b a d}$ in the r.h.s. of

$$
\begin{aligned}
K\left(\alpha_{c}\right)= & H_{F}\left(\alpha_{c}\right)+V_{b a d}\left(\alpha_{c}\right)+\left[R, H_{F}\right]+\left[R, V_{b a d}\right]+\frac{1}{2}\left[R,\left[R, H_{F}\right]\right] \\
& +\frac{1}{2}\left[R,\left[R, V_{b a d}\right]\right]+\ldots+e^{R} V_{g o o d} e^{-R}+e^{R} H_{n s c} e^{-R}
\end{aligned}
$$

(cf. Eq. (2.19) in (Shebeko \& Shirokov, 2001)) by requiring that

$$
\left[H_{F}, R\right]=V_{b a d}
$$

for the operator $R$ of interest.

One should note that unlike the original clothing procedure exposed in (Shebeko \& Shirokov, 2001), (Korda et al., 2007) we eliminate here the bad terms only from $H_{s c}$ interaction in spite of such terms can appear in the nonscalar interaction as well. This preference is relied upon the previous experience (Dubovyk \& Shebeko, 2010) when applying the method of UCTs in the theory of nucleon-nucleon scattering. Now we get the division

$$
H=K\left(\alpha_{c}\right)=K_{F}+K_{I}
$$

with a new free part $K_{F}=H_{F}\left(\alpha_{c}\right) \sim a_{c}^{\dagger} a_{c}$ and interaction

$$
\begin{gathered}
K_{I}=V_{\text {good }}\left(\alpha_{c}\right)+H_{n s c}\left(\alpha_{c}\right)+\left[R, V_{\text {good }}\right] \\
+\frac{1}{2}\left[R, V_{b a d}\right]+\left[R, H_{n s c}\right]+\frac{1}{3}\left[R,\left[R, V_{b a d}\right]\right]+\ldots,
\end{gathered}
$$

where the r.h.s. involves along with good terms other bad terms to be removed via subsequent UCTs described in Subsec. 2.4 of (Shebeko \& Shirokov, 2001) and Sec. 3 of (Korda et al., 2007). In parallel, we have

$$
\mathbf{N} \equiv \mathbf{N}(\alpha)=\mathbf{N}_{F}(\alpha)+\mathbf{N}_{I}(\alpha)=W\left(\alpha_{c}\right) \mathbf{N}\left(\alpha_{c}\right) W^{\dagger}\left(\alpha_{c}\right) \equiv \mathbf{B}\left(\alpha_{c}\right)
$$

or

$$
\mathbf{B}\left(\alpha_{c}\right)=\mathbf{N}_{F}\left(\alpha_{c}\right)+\mathbf{N}_{I}\left(\alpha_{c}\right)+\left[R, \mathbf{N}_{F}\right]+\left[R, \mathbf{N}_{I}\right]+\ldots,
$$

where accordingly the division

$$
\begin{gathered}
\mathbf{N}_{I}=\mathbf{N}_{B}+\mathbf{D}, \\
\mathbf{N}_{B}=-\int \mathbf{x} H_{s c}(\mathbf{x}) d \mathbf{x}=\mathbf{N}_{b a d}+\mathbf{N}_{\text {good }},
\end{gathered}
$$

Eq. (113) can be rewritten as

$$
\begin{aligned}
\mathbf{B}\left(\alpha_{c}\right)= & \mathbf{N}_{F}\left(\alpha_{c}\right)+\mathbf{N}_{b a d}\left(\alpha_{c}\right)+\left[R, \mathbf{N}_{F}\right]+\left[R, \mathbf{N}_{b a d}\right]+\frac{1}{2}\left[R,\left[R, \mathbf{N}_{F}\right]\right] \\
& +\frac{1}{2}\left[R,\left[R, \mathbf{N}_{b a d}\right]\right]+\ldots+e^{R} \mathbf{N}_{g o o d} e^{-R}+e^{R} \mathbf{D} e^{-R} .
\end{aligned}
$$


But it turns out (see the proof of Eq. (3.26) in (Shebeko \& Shirokov, 2001)) that if $R$ meets the condition (109), then

$$
\left[\mathbf{N}_{F}, R\right]=\mathbf{N}_{b a d}=-\int \mathbf{x} V_{b a d}(\mathbf{x}) d \mathbf{x}
$$

so the boost generators in the CPR can be written likely Eq. (110),

$$
\mathbf{N}=\mathbf{B}\left(\alpha_{c}\right)=\mathbf{B}_{F}+\mathbf{B}_{I}
$$

where $\mathbf{B}_{F}=\mathbf{N}_{F}\left(\alpha_{c}\right)$ is the boost operator for noninteracting clothed particles while $\mathbf{B}_{I}$ includes the contributions induced by interactions between them

$$
\begin{gathered}
\mathbf{B}_{I}=\mathbf{N}_{\text {good }}\left(\alpha_{c}\right)+\mathbf{D}\left(\alpha_{c}\right)+\left[R, \mathbf{N}_{\text {good }}\right] \\
+\frac{1}{2}\left[R, \mathbf{N}_{b a d}\right]+[R, \mathbf{D}]+\frac{1}{3}\left[R,\left[R, \mathbf{N}_{b a d}\right]\right]+\ldots
\end{gathered}
$$

One should note that in formulae (111) and (118) we are focused upon the $R$-commutations with the first-eliminated interaction $V_{b a d}$. As shown in (Shebeko \& Shirokov, 2001), the brackets, on the one hand, yield new interactions responsible for different physical processes and, on the other hand, cancel (as a recipe!) the mass and other counterterms that stem from $H_{n s c}\left(\alpha_{c}\right)$ and $\mathbf{D}\left(\alpha_{c}\right)$.

But at this place we will come back to our model with $V_{\text {bad }}=V_{\text {nloc }}, V_{\text {good }}=0$ and $R=R_{\text {nloc }}$ to calculate the simplest commutator $\left[R_{n l o c}, V_{\text {nloc }}\right]$ in which accordingly condition (109) the clothing operator $R_{\text {nloc }}$ is determined by

$$
\left[H_{F}, R_{n l o c}\right]=V_{\text {nloc }} .
$$

From the equation it follows (cf. Appendix A in (Shebeko \& Shirokov, 2001)) that its solution can be given by

$$
R_{n l o c}=\int \frac{d \mathbf{k}}{\omega_{\mathbf{k}}}: F_{b}^{\dagger} R(k) F_{b}: a(k)-\text { H.c. }=\mathcal{R}_{\text {nloc }}-\mathcal{R}_{\text {nloc }}^{\dagger}
$$

with the row $F_{b}^{\dagger}=\left[b(p), d^{\dagger}(p)\right]$ and the column $F_{b}$ (cf. Eq.(A.8) in (Shebeko \& Shirokov, 2001)). The matrix $R(k)$ is composed of the elements

$$
\begin{gathered}
R_{\varepsilon^{\prime} \varepsilon}\left(p^{\prime}, p, k\right)=-\frac{\bar{g}_{\varepsilon^{\prime} \varepsilon}\left(p^{\prime}, p, k\right)}{\omega_{\mathbf{k}}+(-1)^{\varepsilon^{\prime}} E_{\mathbf{p}^{\prime}}-(-1)^{\varepsilon} E_{\mathbf{p}}} \delta\left(\mathbf{k}+(-1)^{\varepsilon^{\prime}} \mathbf{p}^{\prime}-(-1)^{\varepsilon} \mathbf{p}\right) . \\
\left(\varepsilon^{\prime}, \varepsilon=1,2\right)
\end{gathered}
$$

Such a solution is valid if $\mu_{s}<2 \mu_{b}$. In other words, under such an inequality the operator $R_{n l o c}$ has the same structure as $V_{\text {nloc }}$ itself. Then, all we need is to evaluate the commutator $\left[R_{\text {nloc }}, V_{\text {nloc }}\right]$.

For example, our calculations result in the boson-boson interaction operator

$$
\frac{1}{2}\left[R_{n l o c}, V_{n l o c}\right](b b \rightarrow b b)=-\frac{1}{4} \int \frac{d \mathbf{p}_{2}^{\prime}}{E_{\mathbf{p}_{2}^{\prime}}} \int \frac{d \mathbf{p}_{2}}{E_{\mathbf{p}_{2}}} \int \frac{d \mathbf{p}_{1}^{\prime}}{E_{\mathbf{p}_{1}^{\prime}}} \int \frac{d \mathbf{p}_{1}}{E_{\mathbf{p}_{1}}} \delta\left(\mathbf{p}_{1}^{\prime}+\mathbf{p}_{2}^{\prime}-\mathbf{p}_{1}-\mathbf{p}_{2}\right)
$$




$$
\begin{gathered}
\times g_{11}\left(p_{1}^{\prime}, p_{1}, k\right) g_{11}\left(p_{2}^{\prime}, p_{2}, k\right) \\
\times\left\{\frac{1}{\left(p_{1}-p_{1}^{\prime}\right)^{2}-\mu_{s}^{2}}+\frac{1}{\left(p_{2}-p_{2}^{\prime}\right)^{2}-\mu_{s}^{2}}\right\} b_{c}^{\dagger}\left(p_{2}^{\prime}\right) b_{c}^{\dagger}\left(p_{1}^{\prime}\right) b_{c}\left(p_{2}\right) b_{c}\left(p_{1}\right)
\end{gathered}
$$

with $\mathbf{k}=\mathbf{p}_{1}^{\prime}-\mathbf{p}_{1}$ and the respective contribution to $\mathbf{B}_{I}$,

$$
\begin{gathered}
\quad \frac{1}{2}\left[R_{n l o c}, \mathbf{N}_{B}\right](b b \rightarrow b b) \\
=\frac{i}{4} \int \frac{d \mathbf{p}_{2}^{\prime}}{E_{\mathbf{p}_{2}^{\prime}}} \int \frac{d \mathbf{p}_{2}}{E_{\mathbf{p}_{2}}} \int \frac{d \mathbf{p}_{1}^{\prime}}{E_{\mathbf{p}_{1}^{\prime}}} \int \frac{d \mathbf{p}_{1}}{E_{\mathbf{p}_{1}}} \frac{\partial}{\partial \mathbf{p}_{1}^{\prime}} \delta\left(\mathbf{p}_{1}^{\prime}+\mathbf{p}_{2}^{\prime}-\mathbf{p}_{1}-\mathbf{p}_{2}\right) \\
\times g_{11}\left(p_{1}^{\prime}, p_{1}, k\right) g_{11}\left(p_{2}^{\prime}, p_{2}, k\right) \\
\times\left\{\frac{1}{\left(p_{1}-p_{1}^{\prime}\right)^{2}-\mu_{s}^{2}}+\frac{1}{\left(p_{2}-p_{2}^{\prime}\right)^{2}-\mu_{s}^{2}}\right\} b_{c}^{\dagger}\left(p_{2}^{\prime}\right) b_{c}^{\dagger}\left(p_{1}^{\prime}\right) b_{c}\left(p_{2}\right) b_{c}\left(p_{1}\right)
\end{gathered}
$$

In Eqs. (122) and (123) we encounter a covariant (Feynman-like) "propagator"

$$
\frac{1}{2}\left\{\frac{1}{\left(p_{1}-p_{1}^{\prime}\right)^{2}-\mu_{s}^{2}}+\frac{1}{\left(p_{2}-p_{2}^{\prime}\right)^{2}-\mu_{s}^{2}}\right\},
$$

which on the energy shell

$$
E_{\mathbf{p}_{1}}+E_{\mathbf{p}_{1}}=E_{\mathbf{p}_{1}^{\prime}}+E_{\mathbf{p}_{2}^{\prime}}
$$

is converted into the genuine Feynman propagator for the corresponding $\mathrm{S}$ matrix (cf. the first results in (Shebeko \& Shirokov, 2001)).

\subsection{Relativistic interactions between clothed particles in meson-nucleon systems}

Following the same scenario one can derive analytical expressions for separate contributions to the operator

$$
\begin{aligned}
& K_{I} \sim a_{c}^{\dagger} b_{c}^{\dagger} a_{c} b_{c}(\pi N \rightarrow \pi N)+b_{c}^{\dagger} b_{c}^{\dagger} b_{c} b_{c}(N N)\rightarrow N N)+d_{c}^{\dagger} d_{c}^{\dagger} d_{c} d_{c}(\bar{N} \bar{N} \rightarrow \bar{N} \bar{N}) \\
&+b_{c}^{\dagger} b_{c}^{\dagger} b_{c}^{\dagger} b_{c} b_{c} b_{c}(N N N \rightarrow N N N)+\ldots+\left[a_{c}^{\dagger} a_{c}^{\dagger} b_{c} d_{c}+H . c .\right](N \bar{N} \leftrightarrow 2 \pi)+\ldots \\
&+\left[a_{c}^{\dagger} b_{c}^{\dagger} b_{c}^{\dagger} b_{c} b_{c}+\text { H.c. }\right](N N \leftrightarrow \pi N N)+\ldots
\end{aligned}
$$

and, in particular, the operator

$$
\begin{array}{r}
K_{I}^{(2)}=K(N N \rightarrow N N)+K(\bar{N} \bar{N} \rightarrow \bar{N} \bar{N})+K(N \bar{N} \rightarrow N \bar{N})+K(b N \rightarrow b N)+K(b \bar{N} \rightarrow b \bar{N}) \\
+K\left(b b^{\prime} \rightarrow N \bar{N}\right)+K\left(N \bar{N} \rightarrow b b^{\prime}\right)
\end{array}
$$

if one starts with the interactions by Eqs. (50)-(52). It has been done in (Shebeko \& Shirokov, 2001) and (Korda et al., 2007) so many technical details of those derivations can be found 

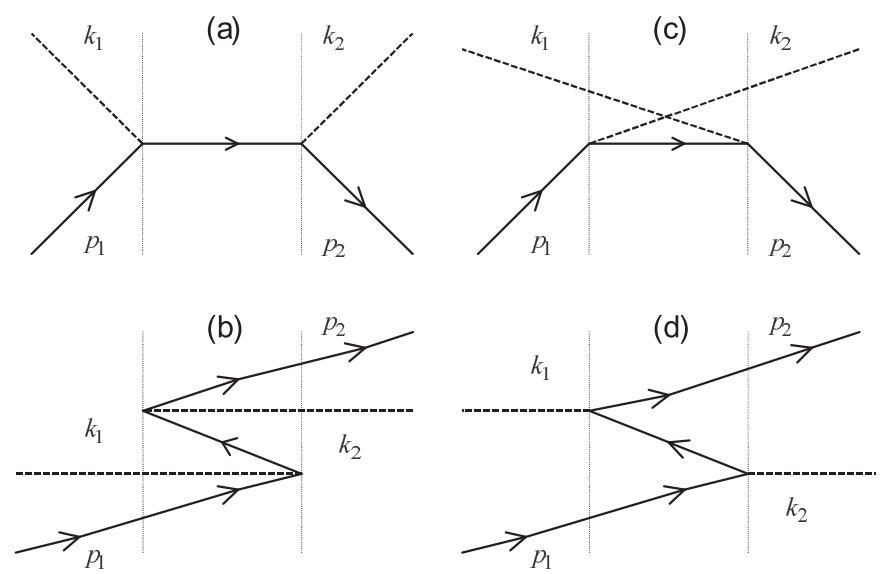

Fig. 1. Different contributions to the $\pi N$ quasipotential.

therein. Note only that the normal ordering is an essential element of them. We will confine ourselves to the two examples that originate from the corresponding commutator $\frac{1}{2}[R, V(b a d)]$.

\subsubsection{Pion-nucleon interaction operator}

As a result, we show the contribution

$$
K(\pi N \rightarrow \pi N)=\int d \vec{p}_{1} d \vec{p}_{2} d \vec{k}_{1} d \vec{k}_{2} V_{\pi N}\left(\vec{k}_{2}, \vec{p}_{2} ; \vec{k}_{1}, \vec{p}_{1}\right) a_{c}^{\dagger}\left(\vec{k}_{2}\right) b_{c}^{\dagger}\left(\vec{p}_{2}\right) a_{c}\left(\vec{k}_{1}\right) b_{c}\left(\vec{p}_{1}\right),
$$

with the following covariant (Feynman-like) form:

$$
\begin{aligned}
& V_{\pi N}\left(\vec{k}_{2}, \vec{p}_{2} ; \vec{k}_{1}, \vec{p}_{1}\right)=\frac{g^{2}}{2(2 \pi)^{3}} \frac{m}{\sqrt{\omega_{\vec{k}_{1}} \omega_{\vec{k}_{2}} E_{\vec{p}_{1} E_{\vec{p}_{2}}}}} \delta\left(\vec{p}_{1}+\vec{k}_{1}-\vec{p}_{2}-\vec{k}_{2}\right) \\
& \begin{aligned}
\bar{u}\left(\vec{p}_{2}\right)\left\{\frac{1}{2}\left[\frac{1}{\hat{p}_{1}+\hat{k}_{1}+m}+\frac{1}{\hat{p}_{2}+\hat{k}_{2}+m}\right] \quad\right. & \left.+\frac{1}{2}\left[\frac{1}{\hat{p}_{1}-\hat{k}_{2}+m}+\frac{1}{\hat{p}_{2}-\hat{k}_{1}+m}\right]\right\} u\left(\vec{p}_{1}\right)
\end{aligned}
\end{aligned}
$$

For brevity, the spin and isospin indices have been omitted.

The corresponding $\pi N$ quasipotential in momentum space is determined by

$$
\tilde{V}_{\pi N}\left(\vec{k}_{2}, \vec{p}_{2} ; \vec{k}_{1}, \vec{p}_{1}\right)=\left\langle a_{c}^{\dagger}\left(\vec{k}_{2}\right) b_{c}^{\dagger}\left(\vec{p}_{2}\right) \Omega|K(\pi N \rightarrow \pi N)| a_{c}^{\dagger}\left(\vec{k}_{1}\right) b_{c}^{\dagger}\left(\vec{p}_{1}\right) \Omega\right\rangle
$$

Graphs in Fig. 1 are topologically equivalent to the well-known time-ordered Feynman diagrams. However, in Schrödinger picture used here, where all events are related to one 
and the same instant $t=0$, such an analogy could be misleading: line directions in Fig. 1 are given with the sole scope to discriminate between nucleon and antinucleon states. Moreover, the energy conservation is not assumed in constructing this and other quasipotentials. Indeed, the coefficients in front of $a_{c}^{\dagger} b_{c}^{\dagger} a_{c} b_{c}$ generally do not fulfill the on-energy-shell condition

$$
E_{\vec{p}_{1}}+\omega_{\vec{k}_{1}}=E_{\vec{p}_{2}}+\omega_{\vec{k}_{2}}
$$

In this connection, the "left" four-vector $s_{1}$ is not necessarily equal to the "right" Mandelstam vector $s_{2}=p_{2}+k_{2}$.

\subsubsection{Nucleon-nucleon interaction operator}

Accordingly (Dubovyk \& Shebeko, 2010) the operator of interest has the following structure:

$$
\begin{gathered}
K(N N \rightarrow N N)=\sum_{b} K_{b}(N N \rightarrow N N), \\
K_{b}(N N \rightarrow N N)=\sum d \vec{p}_{1}^{\prime} d \vec{p}_{2}^{\prime} d \vec{p}_{1} d \vec{p}_{2} V_{b}\left(1^{\prime}, 2^{\prime} ; 1,2\right) b_{c}^{\dagger}\left(1^{\prime}\right) b_{c}^{\dagger}\left(2^{\prime}\right) b_{c}(1) b_{c}(2),
\end{gathered}
$$

where, for example, the c-number matrix $V_{b}$ in the second order in the PS coupling is given by

$$
\begin{gathered}
V_{p s}\left(1^{\prime}, 2^{\prime} ; 1,2\right)=\frac{1}{(2 \pi)^{3}} \frac{m^{2}}{\sqrt{E_{\vec{p}_{1}^{\prime}} E_{\vec{p}_{2}^{\prime}} E_{\vec{p}_{1}} E_{\vec{p}_{2}}}} \delta\left(\vec{p}_{1}^{\prime}+\vec{p}_{2}^{\prime}-\vec{p}_{1}-\vec{p}_{2}\right) v_{p s}\left(1^{\prime}, 2^{\prime} ; 1,2\right), \\
v_{p s}\left(1^{\prime}, 2^{\prime} ; 1,2\right)=\frac{g_{p s}^{2}}{2} \bar{u}\left(\vec{p}_{1}^{\prime}\right) \gamma_{5} u\left(\vec{p}_{1}\right) \frac{1}{\left(p_{1}-p_{1}^{\prime}\right)^{2}-m_{p s}^{2}} \bar{u}\left(\vec{p}_{2}^{\prime}\right) \gamma_{5} u\left(\vec{p}_{2}\right),
\end{gathered}
$$

omitting again the discrete quantum numbers. Here $m_{p s}$ the mass of the clothed pion (its physical value).

Corresponding relativistic and properly symmetrized $N N$ quasipotential

$$
\tilde{V}_{N N}\left(\vec{p}_{1}^{\prime}, \vec{p}_{2}^{\prime} ; \vec{p}_{1}, \vec{p}_{2}\right)=\left\langle b_{c}^{\dagger}\left(\vec{p}_{1}^{\prime}\right) b_{c}^{\dagger}\left(\vec{p}_{2}^{\prime}\right) \Omega\left|K_{N N}\right| b_{c}^{\dagger}\left(\vec{p}_{1}\right) b_{c}^{\dagger}\left(\vec{p}_{2}\right) \Omega\right\rangle
$$

can be written as

$$
\begin{aligned}
& \tilde{V}_{N N}\left(\vec{p}_{1}^{\prime}, \vec{p}_{2}^{\prime} ; \vec{p}_{1}, \vec{p}_{2}\right)=\frac{1}{2} \frac{g_{p s}^{2}}{(2 \pi)^{3}} \frac{m^{2}}{2 \sqrt{E_{\vec{p}_{1}} E_{\vec{p}_{2}} E_{\vec{p}_{1}^{\prime}} E_{\vec{p}_{2}^{\prime}}}} \delta\left(\vec{p}_{1}^{\prime}+\vec{p}_{2}^{\prime}-\vec{p}_{1}-\vec{p}_{2}\right) \\
& \times \bar{u}\left(\vec{p}_{1}^{\prime}\right) \gamma_{5} u\left(\vec{p}_{1}\right) \frac{1}{2}\left\{\frac{1}{\left(p_{1}-p_{1}^{\prime}\right)^{2}-m_{p s}^{2}}\right. \\
& \left.+\frac{1}{\left(p_{2}-p_{2}^{\prime}\right)^{2}-m_{p s}^{2}}\right\} \bar{u}\left(\vec{p}_{2}^{\prime}\right) \gamma_{5} u\left(\vec{p}_{2}\right)-(1 \leftrightarrow 2)
\end{aligned}
$$




\begin{tabular}{|c|c|c|c|}
\hline Meson & & Bonn B & UCT \\
\hline \multirow[t]{3}{*}{$\pi$} & $g_{\pi}^{2} / 4 \pi$ & 14.4 & 13.395 \\
\hline & $\Lambda_{\pi}$ & 1700 & 2500 \\
\hline & $m_{\pi}$ & 138.03 & 138.03 \\
\hline \multirow[t]{3}{*}{$\eta$} & $g_{\eta}^{2} / 4 \pi$ & 3 & 5.0 \\
\hline & $\Lambda_{\eta}$ & 1500 & 1219 \\
\hline & $m_{\eta}$ & 548.8 & 548.8 \\
\hline \multirow[t]{4}{*}{$\rho$} & $g_{\rho}^{2} / 4 \pi$ & 0.9 & 1.2 \\
\hline & $\Lambda_{\rho}$ & 1850 & 1593.0 \\
\hline & $f_{\rho} / g_{\rho}$ & 6.1 & 6.1 \\
\hline & $m_{\rho}$ & 769 & 769 \\
\hline \multirow[t]{3}{*}{$\omega$} & $g_{\omega}^{2} / 4 \pi$ & 24.5 & 17.349 \\
\hline & $\Lambda_{\omega}$ & 1850 & 2494 \\
\hline & $m_{\omega}$ & 782.6 & 782.6 \\
\hline \multirow[t]{3}{*}{$\delta$} & $g_{\delta}^{2} / 4 \pi$ & 2.488 & 5.0 \\
\hline & $\Lambda_{\delta}$ & 2000 & 2169 \\
\hline & $m_{\delta}$ & 983 & 983 \\
\hline \multirow[t]{3}{*}{$\sigma, T=0, T$} & $g_{\sigma}^{2} / 4 \pi$ & $18.3773,8.9437$ & $22.015,5.514$ \\
\hline & $\Lambda_{\sigma}$ & 2000,1900 & 1200,2500 \\
\hline & $m_{\sigma}$ & 720,550 & $691.78,510.62$ \\
\hline
\end{tabular}

Table 1. The best-fit parameters for the two models. The column Potential B (UCT) taken from Table A.1 in (Machleidt, 1989) (obtained by least squares fitting the OBEP values in Table 1 of that survey). All masses are in $\mathrm{MeV}$.

Distinctive feature of potential $\left({ }^{*}\right)$ is the presence of covariant (Feynman-like) "propagator",

$$
\frac{1}{2}\left\{\frac{1}{\left(p_{1}-p_{1}^{\prime}\right)^{2}-\mu^{2}}+\frac{1}{\left(p_{2}-p_{2}^{\prime}\right)^{2}-\mu^{2}}\right\} .
$$

On the energy shell for $N N$ scattering, that is

$$
E_{i} \equiv E_{\vec{p}_{1}}+E_{\vec{p}_{2}}=E_{\vec{p}_{1}^{\prime}}+E_{\vec{p}_{2}^{\prime}} \equiv E_{f},
$$

this expression is converted into the genuine Feynman propagator.

A little part of our numerical results with the best-fit values of the coupling constants $g_{b}$ and cutoff parameters $\Lambda_{b}$ in the meson-nucleon vertices are compared with those by the Bonn group (Machleidt, 1989) in Table 1 and Fig. 2. They labeled by abbreviation UCT have been obtained by solving the partial Lippmann-Schwinger equations (coupled and uncoupled) for the $R$-matrix of the nucleon-nucleon scattering. Details are in (Dubovyk \& Shebeko, 2010).

\subsection{Deuteron properties}

Besides, we would like to outline the basic elements of another our exploration that is in progress. It is the case, where relying upon the available experience of relativistic calculations of the deuteron static moments and the deuteron form factors (see reviews (Bondarenko et al., 2002) and (Garcon \& Van Orden, 2002) and refs. therein) one has to deal with the matrix elements $\left\langle\mathbf{P}^{\prime}, M^{\prime}\left|J^{\mu}(0)\right| \mathbf{P}=0, M\right\rangle$ (to be definite in the laboratory frame). Here the operator 


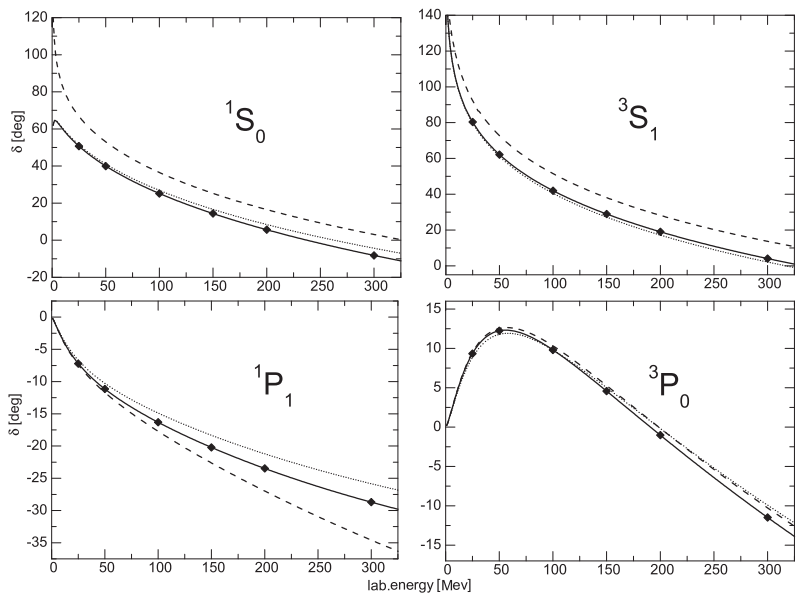

Fig. 2. Neutron-proton phase parameters plotted versus nucleon kinetic energy in lab. system. Solid curves calculated for Potential B. Dashed (dotted) - for UCT potential with Potential B (UCT) parameters from Table 1. The rhombs show original OBEP results.

$J^{\mu}(0)$ is the Nöther current density $J^{\mu}(x)$ at $x=0$, sandwiched between the eigenstates of a "strong" field Hamiltonian $H$ (cf., discussion in Sec. 5 of lecture (Shebeko \& Shirokov, 2000)). In the CPR with $H=K\left(\alpha_{c}\right)$ (Eq. (110)) and $\mathbf{N}=\mathbf{B}\left(\alpha_{c}\right)$ (Eq. (112)) the deuteron state $|\mathbf{P}=0, M\rangle$ $\left(\left|\mathbf{P}^{\prime}=\mathbf{q}, M^{\prime}\right\rangle\right)$ in the rest (the frame moving with the velocity $\left.\mathbf{v}=\mathbf{q} / m_{d}\right)$ meets the eigenvalue equation

$$
P^{\mu}|\mathbf{P}, M\rangle=P_{d}^{\mu}|\mathbf{P}, M\rangle
$$

with the three-momentum transfer $\mathbf{q}$, four-momentum $P_{d}^{\mu}=\left(E_{d}, \mathbf{P}\right), E_{d}=\sqrt{\mathbf{P}^{2}+m_{d}^{2}}, m_{d}=$ $m_{p}+m_{n}-\varepsilon_{d}$ and the deuteron binding energy $\varepsilon_{d}>0$.

We know that such observables as the charge, magnetic and quadrupole moments of the deuteron can be expressed through the matrix elements in question (e.g., within the Bethe-Salpeter (BS) formalism (Bondarenko et al., 2002)) by introducing the corresponding covariant form factors. With the aid of cumbersome numerical methods the latter have been evaluated in terms of the Mandelstam current sandwiched between the deuteron BS amplitudes.

Unlike this, following Shebeko \& Shirokov (2000), we consider the expansion in the $R$-commutators

$$
J^{\mu}(0)=W J_{c}^{\mu}(0) W^{\dagger}=J_{c}^{\mu}(0)+\left[R, J_{c}^{\mu}(0)\right]+\frac{1}{2}\left[R,\left[R, J_{c}^{\mu}(0)\right]\right]+\ldots,
$$

where $J_{c}^{\mu}(0)$ is the initial current in which the bare operators $\{\alpha\}$ are replaced by the clothed ones $\left\{\alpha_{c}\right\}$. Decomposition (134) involves one-body, two-body and more complicated interaction currents, if one uses the terminology customary in the theory of meson exchange currents. Further, to the approximation

$$
K_{I}=K(N N \rightarrow N N) \sim b_{c}^{\dagger} b_{c}^{\dagger} b_{c} b_{c}
$$


and

$$
\mathbf{B}_{I}=\mathbf{B}(N N \rightarrow N N) \sim b_{c}^{\dagger} b_{c}^{\dagger} b_{c} b_{c}
$$

(see, respectively, (122) and (123)) the eigenvalue problem (133) becomes simpler so its solution acquires the form

$$
|\mathbf{P}, M\rangle=\int d \mathbf{p}_{1} \int d \mathbf{p}_{2} D_{M}\left([\mathbf{P}] ; \mathbf{p}_{1} \mu_{1} ; \mathbf{p}_{2} \mu_{2}\right) b_{c}^{\dagger}\left(\mathbf{p}_{1} \mu_{1}\right) b_{c}^{\dagger}\left(\mathbf{p}_{2} \mu_{2}\right)|\Omega\rangle .
$$

In this connection, let us recall the relation

$$
|\mathbf{q}, M\rangle=\exp \left[i \vec{\beta} \mathbf{B}\left(\alpha_{c}\right)\right]|\mathbf{0}, M\rangle
$$

with $\vec{\beta}=\beta \mathbf{n}, \mathbf{n}=\mathbf{n} / n$ and $\tanh \beta=v$, that takes place owing to the property

$$
e^{i \vec{\beta} \mathbf{B}} P^{\mu} e^{-i \vec{\beta} \mathbf{B}}=P^{v} L_{v}^{\mu}(\vec{\beta}),
$$

where $L(\vec{\beta})$ is the matrix of the corresponding Lorentz transformation. Note also that the label $M=( \pm 1,0)$ denotes the eigenvalue of the third component of the total (field) angular-momentum operator in the deuteron center-of-mass (details can be found in (Dubovyk \& Shebeko, 2010)). The $c$-coefficients $D_{M}$ in Eq. (137) are calculated by solving the homogeneous Lippmann-Schwinger equation with the quasipotentials taken from (Dubovyk \& Shebeko, 2010) (see formulae (67)-(69) therein). Numerical results can be obtained either using the angular-momentum decomposition (as in (Dubovyk \& Shebeko, 2010)) or without it.

Several our results are shown in Table 2 and Fig. 3.

\begin{tabular}{|c|c|c|c|}
\hline Parameter & Bonn B & UCT & Experiment \\
\hline$a_{s}(\mathrm{fm})$ & -23.71 & -23.57 & $-23.748 \pm 0.010$ \\
$r_{s}(\mathrm{fm})$ & 2.71 & 2.65 & $2.75 \pm 0.05$ \\
$a_{t}(\mathrm{fm})$ & 5.426 & 5.44 & $5.419 \pm 0.007$ \\
$r_{t}(\mathrm{fm})$ & 1.761 & 1.79 & $1.754 \pm 0.008$ \\
\hline$\varepsilon_{d}(\mathrm{MeV})$ & 2.223 & 2.224 & 2.224575 \\
$P_{D}(\%)$ & 4.99 & 4.89 & \\
\hline
\end{tabular}

Table 2. Deuteron and low-energy parameters. The experimental values are from Table 4.2 of Ref. (Machleidt, 1989).
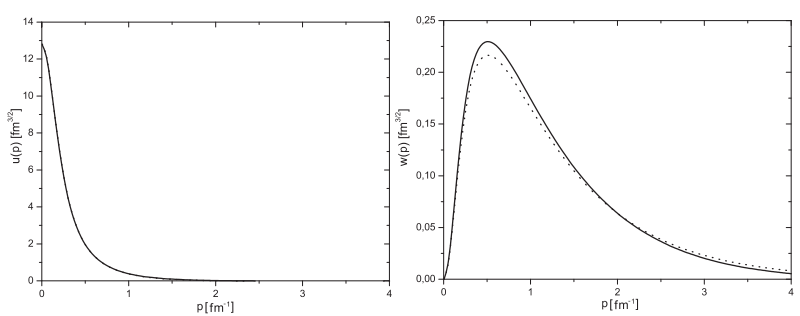

Fig. 3. Deuteron wave functions $\psi_{0}^{d}(p)=u(p)$ and $\psi_{2}^{d}(p)=w(p)$. Solid(dotted) curves for Bonn Potential B (UCT) potential. 
In its turn, the operator (134) being between the two-clothed-nucleon states contributes as

$$
\eta_{c} J^{\mu}(0) \eta_{c}=J_{o n e-b o d y}^{\mu}+J_{t w o-b o d y^{\prime}}^{\mu}
$$

where the operator

$$
J_{\text {one-body }}^{\mu}=\int d \mathbf{p}^{\prime} d \mathbf{p} F_{p, n}^{\mu}\left(\mathbf{p}^{\prime}, \mathbf{p}\right) b_{c}^{\dagger}(\mathbf{p}) b_{c}(\mathbf{p})
$$

with

$$
F_{p, n}^{\mu}\left(\mathbf{p}^{\prime}, \mathbf{p}\right)=e \bar{u}\left(\mathbf{p}^{\prime}\right) F_{1}^{p, n}\left[\left(p^{\prime}-p\right)^{2}\right] \gamma^{\mu}+i \sigma^{\mu v}\left(p^{\prime}-p\right)_{v} F_{2}^{p, n}\left[\left(p^{\prime}-p\right)^{2}\right] u(\mathbf{p})
$$

that describes the virtual photon interaction with the clothed proton (neutron) ${ }^{5}$.

Its appearance follows from the observation, in which the primary Nöther current operator, being between the physical (clothed) states $\left|\Psi_{N}\right\rangle=b_{c}^{\dagger}|\Omega\rangle$, yields the usual on-mass-shell expression

$$
\left\langle\Psi_{p, n}\left(\mathbf{p}^{\prime}\right)\left|J^{\mu}(0)\right| \Psi_{p, n}(\mathbf{p})\right\rangle=F_{p, n}^{\mu}\left(\mathbf{p}^{\prime}, \mathbf{p}\right)
$$

in terms of the Dirac and Pauli nucleon form factors. ${ }^{6}$

By keeping in the r.h.s. of Eq. (140) only the one-body contribution we arrive to certain off-energy-shell extrapolation of the so-called relativistic impulse approximation (RIA) in the theory of e.m. interactions with nuclei (bound systems). In a recent work by Dubovyk and Shebeko the deuteron magnetic and quadrupole moments have been calculated to be submitted to Few Body Systems.

Of course, the RIA results should be corrected including more complex mechanisms of e-d scattering, that are contained in

$$
J_{t w o-b o d y}^{\mu}=\int d \mathbf{p}_{1}^{\prime} d \mathbf{p}_{2}^{\prime} d \mathbf{p}_{1} d \mathbf{p}_{2} F_{M E C}^{\mu}\left(\mathbf{p}_{1}^{\prime}, \mathbf{p}_{2}^{\prime} ; \mathbf{p}_{1}, \mathbf{p}_{2}\right) b_{c}^{\dagger}\left(\mathbf{p}_{1}^{\prime}\right) b_{c}^{\dagger}\left(\mathbf{p}_{1}^{\prime}\right) b_{c}\left(\mathbf{p}_{1}\right) b_{c}\left(\mathbf{p}_{2}\right) .
$$

Analytic (approximate) expressions for the coefficients $F_{M E C}^{\mu}$ stem from the $R$-commutators (beginning with the third one) in the expansion (134), which, first, belong to the class [2.2], as in Eq. (141), and, second, depend on even numbers of mesons involved. It requires a separate consideration aimed at finding a new family of meson exchange currents, as we hope not only for the e-d scattering.

At last, one should note that, as before, we prefer to handle the explicitly gauge-independent (GI) representation of photonuclear reaction amplitudes with one-photon absorption or emission (Levchuk \& Shebeko, 1993). That representation is an extension of the Siegert theorem, in which, the amplitude of interest is expressed through the Fourier transforms of electric (magnetic) field strengths and the generalized electric (magnetic) dipole moments of hadronic system. It allows us to retain the GI in the course of inevitably approximate calculations.

\footnotetext{
${ }^{5}$ In Eqs. (140) $\eta_{c}$ is the projection operator on the subspace $\mathcal{H}_{2 N} \in \mathcal{H}$ spanned on the two-clothed-nucleon states $|2 N\rangle=b_{c}^{\dagger} b_{c}^{\dagger}|\Omega\rangle$

${ }^{6}$ Of course, all nucleon polarization labels are implied here together with necessary summations over them in Eq. (141) and so on
} 


\section{Summary}

We propose a constructive way of ensuring the RI in QFT with cutoffs in momentum space. In contrast to the traditional approach, where the generators of $\Pi$ are determined as the Nöther integrals of the energy-momentum tensor density, we do not utilize the Lagrangian formalism so fruitful in case of local field models. Our purpose is to find these generators as elements of the Lie algebra of $\Pi$ starting from the total Hamiltonian whose interaction density in the Dirac picture includes a Lorentz-scalar part $H_{s c}(x)$. Respectively, the algebraic aspect of the RI as a whole for the present exploration with the so-called instant form of relativistic dynamics is of paramount importance.

In the context, using purely algebraic means the boost generators can be decomposed into the Belinfante operator built of $H_{s c}$ and the operator which accumulates the chain of recursive relations in the second and higher orders in $H_{n s c}$. Thereby, it becomes clear that the Poincaré commutations are not fulfilled if the Hamiltonian does not contain some additional ingredients, which we call the mass renormalization terms, though beyond local field models such a terminology looks rather conventional. The UCT method enables us to determine the corresponding operators including their nonlocal extensions that satisfy the requirements of special relativity and preserve certain continuity with local QFTs.

We see that our approach is sufficiently flexible being applied not merely to local field models including ones with derivative couplings and spins $j \geq 1$. Within the approach all interactions constructed are responsible for physical (not virtual) processes in a given system of interacting fields. Such interactions are Hermitian and energy independent including the off-energy-shell and recoil effects (the latter in all orders of the $1 / c^{2}$ - expansion). In particular, we have managed to build up a new family such interactions in the system of $\pi-, \eta-, \rho-, \omega-, \delta-$ and $\sigma-$ mesons and nucleons. Besides, the interaction operators for processes of the type $N N \rightarrow N N$, $\pi N \rightarrow \pi N$, and $N N \leftrightarrow \pi N N$ are derived on one and the same physical footing.

After constructing the interaction operators in the CPR we express the conventional $S$ matrix through the clothed-particle interactions and states that simplifies the initial field-theoretical task. It becomes possible owing to the isomorphism between the $\alpha$-algebra with the bare vacuum and the $\alpha_{c}$-algebra with the physical vacuum when, first, the requirement iii) is fulfilled and, second, the $R$ - generators of unitary clothing transformations in the Dirac picture come to zero in the distant past and future (see our talk in Durham (Shebeko, 2004)).

In the course of our current work we are trying to understand to what extent the deuteron quenching in flight affects the deuteron electromagnetic form factors. In our opinion, the exposed approach has promising prospects, e.g., in the theory of decaying states (after evident refinements), certainly in quantum electrodynamics and, we believe, in quantum chromodynamics. Such endeavors are under way.

At last, we offer not only a fresh look at constructing the interactions in question but also a nonstandard renormalization procedure in relativistic quantum field theory. In this context, let us remind the prophetic words by Dirac (Dirac, 1963): "I am inclined to suspect that renormalization theory is something that will not survive in the future, and the remarkable agreement between theory and experiment should be looked on as a fluke". 


\section{Acknowledgements}

This exposition is dedicated to the memory of Mikhail Shirokov, the excellent scientist whose contribution to the subfield is difficult to be overestimated. I am very grateful to Luciano Canton, Evgenyi Dubovik, Pavel Frolov and Vladimir Korda for our fruitful collaboration.

\section{References}

Albeverio, S. (1973). Scattering theory in a model of quantum fields, I. J. Math. Phys., 18, 1800-1816.

Arnous, E.; Heitler W. ; Takahashi Y. (1960). On a convergent non-local field theory, Nuovo. Cim., 16, 671-682.

Bakker, B.L.G. (2001). Forms of relativistic dynamics, In:Lecture Notes in Physics, Latal, H. and Schweiger, W. (ed.), Springer-Verlag, pp. 1-54.

Belinfante, F.J. (1940). On the covariant derivative of tensor-undors, Physica, 7, 305-324.

Bondarenko, S. G.; Burov, V. V.; Molochkov, A. V.; Smirnov, G. I.; Toki, H. (2002).

The Bethe-Salpeter approach with the separable interaction for the deuteron, Prog. Part. Nucl. Phys., 48, 449-535.

Chandler, C. (2003). Relativistic scattering theory with particle creation. Contribution to the 17th International IUPAP Conference on Few Body Physics (Durham, USA) and private communication.

Dirac, P.A.M. (1949). Forms of relativistic dynamics, Rev. Mod. Phys., 21, 392-399.

Dirac, P.A.M. (1963). The evolution of the physicist's picture of nature, Sci. Amer., 208, 45-53.

Dubovyk, E.A.; Shebeko, A.V. (2010). The method of unitary clothing transformations in the theory of nucleon-nucleon scattering, Few-Body Syst., 48, 109-142.

Eckmann, J.-P. (1970). A model with persistent vacuum, Commun. Math. Phys., 18, 247-264.

Efimov, G.V. (1985). Problems of the quantum theory of nonlocal interactions, Nauka, Moscow, (in Russian).

Foldy, L.L. (1961). Relativistic particle systems with interactions, Phys. Rev., 122, 275-288.

Friar, J.L. (1975). Relativistic effects on the wave function of a moving system, Phys. Rev. C 12, 695-698.

Friedrichs, K. (1953). Mathematical Aspects of the Quantum Theory of Field, Interscience, New York.

Glöckle, W.; Müller, L. (1981). Relativistic theory of interacting particles, Phys. Rev. C 23, 1183-1195.

Garçon, M.; Van Orden, J.W. (2002). The deuteron: structure and form factors. Adv. Nucl. Phys., 26, 293-378.

Greenberg, O.; Schweber, S. (1958). Clothed particle operators in simple models of quantum field theory, Nuovo Cim., 8, 378-406.

Guillot, J.C.; Jaus, W.; O'Raifeartaigh, L. (1965). A survey of the Heitler-Arnous non-local field theory, Proc. R.I.A., 64, 93-105.

Heinzl, T. (2001). Light-cone quantization foundations and applications, In:Lecture Notes in Physics, Latal, H. and Schweiger, W. (ed.), Springer-Verlag, pp. 55-78.

Keister, B.D.; Polyzou, W.N. (1991). Relativistic Hamiltonian dynamics in nuclear and particle physics, Adv. Nucl. Phys., 20, 225-479.

Kita, H. (1966). A non-trivial example of a relativistic quantum theory of particles without divergence difficulties, Prog. Theor. Phys., 35, 934-959. 
Kita, H. (1968). Another convergent, relativistic model theory of interacting particles. A relativistic version of a modified Lee model, Prog. Theor. Phys., 39, 1333-1360.

Kobayashi, M.; Sato, T.; Ohtsubo, H. (1997). Effective interactions for mesons and baryons in nuclei, Progr. Theor. Phys., 98, 927-941.

Korda, V.Yu.; Shebeko, A.V. (2004). Clothed particle representation in quantum field theory: Mass renormalization, Phys. Rev. D 70, 085011.

Korda, V.Yu.; Canton, L.; Shebeko, A.V. (2007). Relativistic interactions for the meson-two-nucleon system in the clothed-particle unitary representation, Ann. Phys., 322, 736-768.

Krüger, A.; Glöckle, W. (1999). Approach towards N-nucleon effective generators of the Poincaré group derived from a field theory, Phys. Rev. C 59, 31919-1929.

Levchuk, L.G.; Shebeko, A.V. (1993). On a generalization of Siegert's theorem. A corrected result, Phys. At. Nuclei, 56, 227-229.

Machleidt, R. (1989). The meson theory of nuclear forces and nuclear structure, Adv. Nucl. Phys., 19, 189-376.

Melde, T.; Canton, L.; Plessas, W. (2009). Structure of meson-baryon interaction vertices, Phys. Rev. Lett., 102, 132002.

Nelson, E. (1964). Interaction of nonrelativistic particles with a quantized scalar field, J. Math. Phys., 5, 1190-1197.

Okubo, S. (1954). Diagonalization of Hamiltonian and Tamm-Dancoff equation, Prog. Theor. Phys., 12, 603-608.

Schweber, S.S. (1961). An Introduction to Relativistic Quantum Field Theory, Row, Peterson, New York.

Schwinger, J. (1962). Non-abelian gauge fields. Relativistic invariance, Phys. Rev., 127, 324-330.

Shebeko, A.V. (1990). Local analog of the Siegert theorem for covariant description of electromagnetic interactions with nuclei. Sov. J. Nucl. Phys., 52, 970-975.

Shebeko, A.V. (2004). The S matrix in the method of unitary clothing transformations, Nucl. Phys. A 737, 252-254.

Shebeko, A.V. and Shirokov, M.I. (2000).Clothing procedure in relativistic quantum field theory and its applications to description of electromagnetic interactions with nuclei (bound systems), Progr. Part. Nucl. Phys., 44, 75-86.

Shebeko, A.V. and Shirokov, M.I. (2001). Unitary transformations in quantum field theory and bound states, Phys. Part. Nuclei, 32, 31-95.

Shebeko, A.; Frolov, P. (2011). A possible way for constructing the generators of the Poincaré group in quantum field theory, to appear in : Few Body Systems

Shirokov, M.I. (2002). Relativistic nonlocal quantum field theory, Int. J. Theor. Phys., 41, $1027-1041$.

Stefanovich, E. (2001). Quantum field theories without infinities, Ann. Phys., 292, 139.

Weinberg, S. (1995). The Quantum Theory of Fields, University Press, Cambridge, Vol.1.

Wentzel, G. (1949). Quantum Theory of Fields, Interscience, New York. 


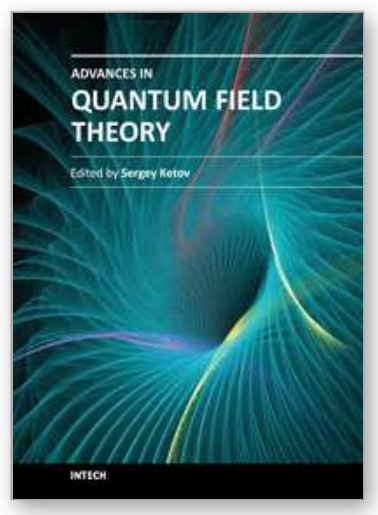

\author{
Advances in Quantum Field Theory \\ Edited by Prof. Sergey Ketov
}

ISBN 978-953-51-0035-5

Hard cover, 230 pages

Publisher InTech

Published online 03, February, 2012

Published in print edition February, 2012

Quantum Field Theory is now well recognized as a powerful tool not only in Particle Physics but also in Nuclear Physics, Condensed Matter Physics, Solid State Physics and even in Mathematics. In this book some current applications of Quantum Field Theory to those areas of modern physics and mathematics are collected, in order to offer a deeper understanding of known facts and unsolved problems.

\title{
How to reference
}

In order to correctly reference this scholarly work, feel free to copy and paste the following:

A. V. Shebeko (2012). The Method of Unitary Clothing Transformations in Quantum Field Theory: Applications in the Theory of Nuclear Forces and Reactions, Advances in Quantum Field Theory, Prof. Sergey Ketov (Ed.), ISBN: 978-953-51-0035-5, InTech, Available from: http://www.intechopen.com/books/advances-in-quantumfield-theory/the-method-of-unitary-clothing-transformations-in-quantum-field-theory-applications-in-the-theoryof

\section{INTECH}

open science | open minds

\author{
InTech Europe \\ University Campus STeP Ri \\ Slavka Krautzeka 83/A \\ 51000 Rijeka, Croatia \\ Phone: +385 (51) 770447 \\ Fax: +385 (51) 686166 \\ www.intechopen.com
}

\author{
InTech China \\ Unit 405, Office Block, Hotel Equatorial Shanghai \\ No.65, Yan An Road (West), Shanghai, 200040, China \\ 中国上海市延安西路65号上海国际贵都大饭店办公楼 405 单元 \\ Phone: +86-21-62489820 \\ Fax: +86-21-62489821
}


(C) 2012 The Author(s). Licensee IntechOpen. This is an open access article distributed under the terms of the Creative Commons Attribution 3.0 License, which permits unrestricted use, distribution, and reproduction in any medium, provided the original work is properly cited. 